\title{
Evaluation of Grid Modification Methods for On- and Off-Track Sonic Boom Analysis
}

\author{
Sudheer N. Nayani ${ }^{1}$ \\ Analytical Services \& Materials, Inc., Hampton, VA 23666 \\ and \\ Richard L. Campbell ${ }^{2}$ \\ NASA Langley Research Center, Hampton VA 23681
}

\begin{abstract}
Grid modification methods have been under development at NASA to enable better predictions of low boom pressure signatures from supersonic aircraft. As part of this effort, two new codes, Stretched and Sheared Grid - Modified (SSG) and Boom Grid (BG), have been developed in the past year. The CFD results from these codes have been compared with ones from the earlier grid modification codes Stretched and Sheared Grid (SSGRID) and Mach Cone Aligned Prism (MCAP) and also with the available experimental results. NASA's unstructured grid suite of software TetrUSS and the automatic sourcing code AUTOSRC were used for base grid generation and flow solutions. The BG method has been evaluated on three wind tunnel models. Pressure signatures have been obtained up to two body lengths below a Gulfstream aircraft wind tunnel model. Good agreement with the wind tunnel results have been obtained for both on-track and off-track (up to 53 degrees) cases. On-track pressure signatures up to ten body lengths below a Straight Line Segmented Leading Edge (SLSLE) wind tunnel model have been extracted. Good agreement with the wind tunnel results have been obtained. Pressure signatures have been obtained at 1.5 body lengths below a Lockheed Martin aircraft wind tunnel model. Good agreement with the wind tunnel results have been obtained for both on-track and off-track (up to 40 degrees) cases. Grid sensitivity studies have been carried out to investigate any grid size related issues. Methods have been evaluated for fully turbulent, mixed laminar/turbulent and fully laminar flow conditions.
\end{abstract}

\section{Nomenclature}

$\mathrm{H} / \mathrm{L}=$ distance below the model normalized by model length (or body length)

$\mathrm{L} \quad=$ reference model length, inches

$\mathrm{M}_{\infty} \quad=$ free-stream Mach number

$\Delta \mathrm{p}_{\infty} / \mathrm{p}=\left(\mathrm{p}-\mathrm{p}_{\infty}\right) / \mathrm{p}_{\infty}$

$\mathrm{p}=$ static pressure

$\mathrm{p}_{\infty} \quad=$ free-stream static pressure

$\mathrm{Re}_{\mathrm{L}} \quad=$ Reynolds number based on reference length

$\mathrm{S} \quad=$ radial distance

SST = Shear Stress Transport turbulence model

$x=$ distance in the stream-wise direction measured from the tip of the model nose, inches

\footnotetext{
${ }^{1}$ Senior Scientist, CFD Group, 107 Research Drive, AIAA Senior Member.

2 Senior Research Engineer, Configuration Aerodynamics Branch, MS 499, AIAA Associate Fellow.
} 


$\begin{array}{ll}\mathrm{X} / \mathrm{L} & =\text { non-dimensionalized distance in the stream-wise direction measured from the tip of the model nose } \\ \mathrm{y}^{+} & =\text {first viscous layer spacing parameter } \\ \alpha & =\text { angle of attack, degrees } \\ \delta & =\text { average axial distance between points along the junction between the outer boundary } \\ & \text { and symmetry plane } \\ & =\text { radial angle used in grid generation }\end{array}$

\section{Introduction}

There has been a revival of interest in supersonic business jets as evidenced by the efforts at companies like Gulfstream, Aerion etc. Though great strides have been made in the areas of structures and aerodynamics, the main issue still remains relative to the reduction of sonic boom over-pressure on the ground to a level that would allow supersonic flight over land. One of the other focal areas in the NASA Fundamental Aeronautics Program Supersonics Project is supersonic cruise efficiency. The goal in this area is to develop a better understanding of the flow physics that affect the performance of a supersonic aircraft using computational and experimental studies. This understanding will then be used to develop and apply tools and technologies that will enable the design of efficient aircraft that will also meet constraints such as low sonic boom levels.

Grid generation and grid refinement/alignment methods play a critical role in the prediction of boom signatures. Conventional isotropic refinement methods like CRISP ${ }^{1}$ add more points in the vicinity of shocks and can produce better defined shocks in those regions, but do not necessarily allow the shocks to propagate accurately further into the flow field. Also, CRISP does not align the faces with shocks/expansions so that the high gradients occur across the faces. This seems to imply that close grid spacing is required normal to the shocks and compression waves to adequately resolve the gradients, but a coarse grid spacing is needed in the propagation direction to minimize the number of cells traversed to reach the mid-field.

\section{Grid Generation and Computational Fluid Dynamics (CFD) Analysis}

\section{Initial Grid Generation}

This computational fluid dynamics study used the NASA Tetrahedral Unstructured Software System (TetrUSS ${ }^{2}$ ) for all of the initial grid generation as well as the computations. This CFD suite, created and maintained at NASA Langley Research Center, includes a geometry setup tool called Gridtool, an unstructured grid generation program called VGRID, a postprocessor named POSTGRID, and the flow solver USM3D. The Computer-aided design (CAD) model of the configuration (typically an IGES file) is read into the pre-processing code GridTool $^{3}$ where the curves and patches on the surfaces and far-field are then defined.

The initial layout and sizing of the line sources used by VGRID to control grid density are created automatically using the AUTOSRC code ${ }^{4}$. Line sources have been sufficiently stretched at the required locations (e.g. sharp corners) to avoid the occurrence of any flat cells. In addition to these basic line sources, for cases involving sonic boom or powered nacelle calculations, AUTOSRC will add a volume source to increase the field grid density in the region of interest. The location, size and grid spacing parameters are determined based on the configuration geometry, but also vary depending on the code used to modify the initial grid for boom calculations. Further details on these variations will be included in the discussions of the grid modification methods in the following sections. For some configurations, additional sources were manually provided to locally increase grid density for improved accuracy (e.g. a cylindrical volume source around the wing) or to enable grid generation to proceed in a complex region (e.g., flap edges).

VGRID is a tetrahedral unstructured grid generation program that can be run interactively or in batch mode. The grids produced by VGRID are suitable for computing Euler or Navier-Stokes flow solutions. The grid spacing is related to the strength of user-defined sources placed in the domain. The methodology is based on the AdvancingLayers method (ALM) ${ }^{5}$ and the Advancing-Front method (AFM) ${ }^{6}$. Both techniques are based on marching processes in which tetrahedral cells are grown on an initial triangular boundary mesh and gradually form in the field around the geometry. Once the advancing front process is completed in VGRID, an additional post-processing step is 
required using POSTGRID to close any open pockets in the grid and improve grid quality.

\section{Shearing and Stretching Methods}

Clustering surface grid points near the leading edge of a wing to resolve both the surface curvature as well as the flow acceleration is a common example of the knowledge-based approach used in both unstructured and structured grid generation. Control of the field grid characteristics is more difficult, especially for unstructured grids and even for structured grids around complex configurations. The unstructured grid generation code VGRID ${ }^{7}$ utilizes a variety of 'source' types to control field grid placement. In particular, volume sources have been successfully used to control grid density and orientation in a number of applications ${ }^{4}$. Although this approach has been used to produce stretched and sheared grids for sonic boom analysis ${ }^{8}$, the major drawbacks were that it proved to be a fairly userintensive process and had the disadvantage of having to be repeated for each new Mach number or angle of attack for a configuration.

The Stretched and Sheared Grid (SSGRID) $)^{4,8}$ code uses an a priori grid adaptation approach, where the initial grid is clustered and oriented based on general flow characteristics that are known in advance of running the flow solver. The SSGRID code avoids the earlier drawbacks by shearing and stretching the original grid to align the cell faces with the free-stream Mach angle as well as reducing the number of points required to reach a given distance from the aircraft. The initial grid is typically generated with VGRID using relatively large field grid spacing, except for a region of dense grid underneath the vehicle that is created using a vertical cylindrical volume source. If the grid shearing begins too close to the body, it might cause the cells to intersect the wings or other components, or it could distort the structure of the boundary layer grid for viscous cases. To avoid this potential problem, an inner cylinder parallel to the body axis and just outside the wing tip is defined within which no grid modification occurs (see figure 1). If a circular cylinder is used to define this region, the stretching would typically begin farther from the bottom of the fuselage of the aircraft than it needs to be and could allow unnecessary dissipation to occur before the sheared grid is reached. To remedy this, a variable inner cylinder radius is used based on the keel line. The keel line, primary inner cylinder radius, and variable radius are all automatically determined in SSGRID based on the aircraft geometry and initial grid characteristics.

The grid points between the inner cylinder and outer boundary are first stretched in radial directions to a userspecified distance and then sheared conically to match the free-stream Mach angle. This aligns the stretch direction with the shocks and expansions. Because the flow gradients are small in the stretch direction, the larger cell size allows these pressure waves to propagate to the mid-field with less dissipation than with the original grid. The grid stretching and shearing in SSGRID will generally produce some negative volume cells, so the grid is run through the ADV (ADaptation for Volume grid) grid post-processing code ${ }^{4}$ to repair these cells. The entire process is automated and typically takes less than a minute to create a grid for a prescribed Mach number and an angle-of-attack. The primary user-supplied inputs are the free-stream Mach number, angle of attack, reference body length, and the desired location for the stretched outer boundary in body lengths below the aircraft.

The radial approach to stretching in SSGRID should provide fairly good results at off-track angles as well as directly under the model. However, the radial method as implemented also had some limitations for certain types of configurations and relative to the extent of stretching before the ADV negative cell clean up method failed.

To address these limitations, the Stretched and Sheared Grid - Modified (SSG) method was developed from the SSGRID code. Initially, the primary difference between the two codes was that the SSG method did the shearing and stretching only in the vertical direction and only below the configuration. This approach has the advantage of allowing the origin of the stretching to be extended above the fuselage centerline in the region beyond the aft end of the fuselage to better propagate flow features emanating from the empennage or engine components. It also provides a better grid alignment angle for pressure waves originating from outboard components such as the wing tips relative to the centerline boom signature. Experience indicates the SSG method creates fewer negative volume grid cells that need to be removed in post-processing while maintaining good on-track boom signature quality. This approach provided a limited ( 15 degree) off-track signature capability; however, as interest increased in a range of signature angles from centerline to 45 degrees or more off-track, it became necessary to modify the shearing approach. A span-wise component was added to the shearing, with the stream-wise component adjusted to approximate a conical distribution from the keel line. 


\section{Prism Extrusion Methods}

While the SSGRID and SSG methods have been fairly successful in their own right, they have also played a key role as pre-processors for an adjoint-based grid adaptation method that utilizes unstructured grids ${ }^{9}$. Their successes notwithstanding, the occasional problem with grid clean up prompted a search for a more robust approach to sonic boom grid generation. An alternative approach that grew out of the shear/stretch philosophy is the Mach Cone Aligned Prism (MCAP) method ${ }^{10}$.

The MCAP method applies the shearing and stretching principles to a "collar" grid that is added to an internal "core" grid. The collar grid is generated by extruding prisms from the triangulated outer boundary faces of the core grid, with the prism edges being sheared and stretched radially along Mach angles. While this approach does not necessarily eliminate the development of negative cells in the collar grid because of the faceted character of the core grid outer boundary, it typically allows larger gridding distances to be reached before they occur. In the authors' limited experience with MCAP, the grid generation process generally requires about an order of magnitude more time than the SSG process, but it still typically takes less than an hour. Because new cells are being added, the MCAP grids also tend to be larger than ones from SSG, but the grid quality and convergence characteristics are greatly improved. The grid generation process also slows down due to the fact that the MCAP method converts the VGRID format files to "AIRPLANE' 11 unstructured Euler flow solver format files, adds a collar and translates them back again to the VGRID format. More detail on the method as well as a number of verification examples can be found in reference 10 .

In spite of the improved grid quality, it has been noted that SSG provided better detail in some regions of boom signatures than did the MCAP methodology. In an effort to explore this discrepancy as well as reduce the time required for generating the collar grid, an existing grid-extrusion code (Q2D) $)^{12}$ was modified to incorporate the collar grid capability.

The Q2D code was originally developed during a joint effort with Lockheed-Martin in the Air Force Transonic Efficient Mobility Planform Optimization (TEMPO) ${ }^{12}$ program. The purpose of the code was to generate 'quasi-2D' unstructured grids that were one cell wide, thus allowing advanced airfoil concepts to be evaluated while reducing the computational time by as much as a factor of 40 relative to the normal practice of generating a grid on a constant-chord wing segment.

The Q2D process begins with the generation of a 2-D unstructured grid around an airfoil using the VGRID grid generator, and then prisms are extruded from the triangular faces in the span-wise direction from this symmetry plane. The prisms are then split into tetrahedral cells using a nodal numbering system based on point layers, with the appropriate cell sides saved as boundary faces. This approach is extremely efficient, creating grids with tens of millions of cells in typically less than a minute. The Q2D code has recently been extended to create grids ranging from a one-cell wide 'pie-slice' (2-D axisymmetric) to a full 360-degree grid for axisymmetric bodies as well as circular inlets and nozzles for aircraft engines. For these cases, all of the cells in the final grid are generated by the Q2D code. Since the collar grid approach adds points, faces and cells to an existing 3-D grid, it was decided to create a separate version of Q2D, referred to as the Boom Grid (BG) code.

The BG code utilizes the same methodology as Q2D for creating and splitting the prisms. As with MCAP, the prisms are extruded from the faces of the core grid outer boundary. In order to take full advantage of the BG method, the AUTOSRC code was modified to adjust the original cylindrical outer boundary size and location, and a new approach to locating the boom volume source was defined. The code first determines the location of the lowest point on the fuselage keel line (keel min $\mathrm{z}$ in figure 2) and the wing tip. A new radius for the outer boundary is defined so that it passes through points that are two percent of the body length below these locations (A and B in figure 2). If the resulting radius is greater than one body length, the distance between point $\mathrm{A}$ and keel min $\mathrm{z}$ is increased until the one body length limit for the radius is reached. This approach attempts to minimize the amount of unstretched/unsheared grid through which a pressure wave must travel while keeping the outer boundary size reasonable. 
For the BG approach, the primary factor affecting the field grid density for propagation of the sonic boom signature is the spacing of the core grid along the outer boundary. In order to achieve a high point density below the configuration without adding too many points in the rest of the core grid, a horizontal-axis cylindrical volume source is defined below the configuration with twice the radius of the core grid outer boundary and its outer cylinder passing through points A and B (dashed magenta line in figure 2). The resulting core grid inflow plane (figure 3) and symmetry plane in the nose region (figure 4) indicate that the desired grid spacing characteristics were achieved.

The prism extrusion process in the BG code begins with reading the core grid and identifying the outer boundary triangular faces. The points on these faces are then assigned to layers starting from the most forward point at the lower junction with the symmetry plane. This scheme allows the prisms to be generated and split into tetrahedral cells with consistent face definitions, thus eliminating any need to check and re-orient faces. The prism edges are extruded from the boundary face points as shown in the left half of figure 5 . The first layer radial distance $\left(\mathrm{s}_{1}\right)$ is determined based on a user-specified factor (S1) times the average axial distance between points along the junction between the outer boundary and symmetry plane, $\delta$.

$$
\mathrm{s}_{1}=\mathrm{S} 1 * \delta
$$

This spacing is then increased by another specified factor (R1) for subsequent layers

$$
\mathrm{s}_{\mathrm{i}}=\mathrm{s}_{\mathrm{i}-1} * \mathrm{R} 1
$$

until a maximum spacing limit (S2) is reached, with additional layers maintaining this maximum spacing. The initial distance, growth rate and maximum distance were evaluated and recommended values will be discussed in a later section of this paper.

As can be seen in the right side of figure 5, the reference point for determining the radial direction for the extrusion of the prism edges in BG is not constrained to be at the center of the outer boundary cylinder. As the goal in creating the outer grid is to align the grid as closely as possible with the Mach lines emanating from the configuration, it was observed that, for example, placing the radial reference point at the fuselage center would best align the grid with flow features generated by that component. It was found that an approach that began with the reference point near the nose for points on the outer boundary near the symmetry plane, and then shifting the reference point toward the center of the outer boundary cylinder for points at the maximum width of the outer boundary, could produce both a clustering and alignment of the grid near a desired track angle for the signature. For cases looking at on-track signatures (directly below the aircraft), the reference point moves immediately from near the nose to the center of the outer boundary cylinder. This is illustrated in figure 6, where the blue line labeled "zpr line" is the path traced by the z-coordinate of the radial angle (phi) reference point. The red lines are representative radial lines for prism extrusion. The resulting inflow plane for the new outer grid is shown in figure 7 with the radial lines extended to the new outer boundary. The grid is most densely clustered directly below the aircraft, which should optimize the propagation of features in the on-track signature generated by components close to the centerline of the aircraft, but may lead to some degradation for ones originating from outboard regions such as the wing tip. Similarly, the accuracy of off-track signatures would likely be somewhat reduced, although the clustering is still fairly dense for off-track angles up to 30 degrees or so.

When off-track signatures are desired, the z-location of the reference point is more slowly moved toward the center of the outer boundary cylinder (see figure 8). This has the effect of skewing the grid lines more outboard for a given initial location on the core grid outer boundary and provides better alignment and clustering for the desired off-track angle (shown by the black line in figure 9). Note that the clustering is still dense above the desired angle, which should improve the propagation of features generated above and/or outboard of the nose. An initial evaluation of using a single on- or off-track grid for computing boom signatures at multiple track angles was performed and will be discussed in the Results section.

As noted early, although the prism extrusion methods are generally more robust than the original grid stretching and shearing approaches, the triangular faceting of the original outer boundary grid can still lead to highly-sheared "sliver" and/or negative-volume cells as the outer radius expands and the ratio of circumferential-to-axial distances 
on faces increases. The BG method includes some coding that checks for and attempts to repair these cells as the grid is generated, allowing signatures to be obtained at greater distances than previous approaches.

\section{Flow Solution Methodology}

The USM3D code $^{13}$ is a cell-centered, finite-volume Navier-Stokes flow solver that uses various flux-differencing schemes to compute inviscid flux quantities across the faces of the tetrahedral cells. The Harten, Lax, van LeerContact (HLLC) scheme was used for the present study. Several options for turbulent closure are available: the oneequation Spalart-Allmaras (S-A) mode ${ }^{14}$ (with and without a wall function), and several two-equation models, including Menter's Shear Stress Transport (SST) model. ${ }^{15}$ The minmod limiter was used for all of the simulations presented in this paper. For cases involving turbulent flow, the SST turbulence model was invoked.

In addition to the turbulence model options mentioned above, USM3D has been modified by Pandya ${ }^{16}$ to include the capability for specifying the location of boundary layer transition on any given component. This location can be directly specified to match a known position (e.g., the location of a transition strip on a wind tunnel model) or may be computed using a transition prediction method such as MATTC ${ }^{17}$. For the cases in this study that involve transition (referred to as "mixed flow"), the location was directly specified and the "forced laminization" option discussed in reference 16 was used.

The parallel version of the flow solver was run at the NASA Advanced Supercomputing (NAS) facility on large Linux clusters at NASA Ames Research Center. Three different configurations were analyzed in this study, a Gulfstream configuration, a Lockheed Martin configuration, and a Straight Line Segmented Leading Edge (SLSLE) configuration. A flow solution on the Gulfstream configuration took about $1150 \mathrm{CPU}$ hours when 96 processors were used for a typical grid size of 35 million cells. The flow solution was run for 12000 iterations for the required convergence. On the Lockheed Martin configuration, a flow solution took about 1850 CPU hours when 96 processors were used for a typical grid size of 29.4 million cells. The flow solution was run for 27000 iterations for the required convergence. For the Gulfstream and Lockheed configurations, Westmere nodes of Pleiades supercomputers were used. On the SLSLE configuration, a flow solution took about 2900 CPU hours when 512 processors were used for a typical grid size of 68.8 million cells. The flow solution was run for 20000 iterations for the required convergence. SLSLE model flow solutions were run using Sandybridge nodes on the Pleiades supercomputer.

\section{Results}

Results are provided for the various boom gridding methods applied to several configurations. For each configuration, the boom sourcing approaches are illustrated, followed by plots of the boom signature results for the different gridding methods along with experimental data when available. In addition, some results from studies looking at grid size, boundary layer state and BG input parameters have also been included.

\section{Gulfstream Configuration}

The Gulfstream boom model used in the present study is shown in Figure 10. This configuration was tested in the 4foot Supersonic Unitary Plan wind tunnel (UPWT) at NASA Langley Research Center. The model has a blade sting starting near the center of the body and moving away to the far-field, in the back. All the CFD cases presented here were run with a free-stream Mach number of 1.56, angle of attack of 0.256 degrees and a Reynolds number of 3.86 million based on a reference length of 13.2 inches. CFD results have been compared with two sets of experimental data. The data designated as 'fixed transition' is a case where the boundary layer on the wind tunnel model had transition disks applied as shown in Figure 11 to trip the boundary layer. The data designated as 'free transition' is for the case where the boundary layer was not tripped.

Figure 12a and 12b show the sourcing used to generate the base grid for the SSGRID method. Line sources are used for the configuration and a vertical axis cylindrical source is used for the boom propagation region. A rectangular outer box has been used for the far-field boundary. The outer boundary is roughly a body length away from the surface in all three directions. Figure 12c shows the surface base grid and Figure 12d shows the final grid in the 
symmetry plane after it has been sheared and stretched in the Mach angle direction. Note that the grid has been stretched above as well as below the model. Figure 13 shows the grid generation sequence for the SSG method. As shown in Figures 13a and 13b, in this method a horizontal cylindrical source is placed around the model, making sure that it covers the entire configuration except the sting. The outer box has a radius equal to the model body length with the center at the tip of the model nose. Figure 13c shows the surface base grid and Figure 13d shows the grid in the symmetry plane after shearing and stretching. Comparing Figure 12d and 13d, it can be seen that the SSG method has stretched the grid only below the body. Figure 14 details the grid generation procedure for the MCAP method. In this case, the outer boundary is just big enough to enclose the body and the sting. Two cylindrical sources are used as shown, one around the wing and a short vertical source from the body to the outer boundary. Figure 14c is the base grid generated by VGRID. This acts as an inner grid and as shown in Figure 14d, a collar grid is added to the inner grid and stretched in all directions.

Figure 15 details the grid generation sequence in the BG method on a Gulfstream configuration. In this method, the body is moved down as close as possible to the outer boundary as shown in Figure 15b. The cylindrical source around the wing is not used. The AUTOSRC program places a cylindrical source of a larger diameter with its center offset from the symmetry plane. The placement and diameter of this boom source ensures the required grid resolution for both on-track and off-track conditions. Figure 15c shows the surface grid before the collar is added and Figure 15d shows the stretched and sheared final grid after the collar has been added in the symmetry plane. Three grids have been generated on the Gulfstream configuration, one with on-track grid clustering and the other two with grid clustering at off-track angles of 35 and 53.4 degrees, respectively. For the on-track pressure signature, the grid with on-track clustering was used. For the off-track signatures of 25.5 and 35 degrees, the grid with grid clustering at 35 degrees was used. For the off-track signature of 53.4 degrees, the grid with grid clustering at 53.4 degrees was used.

Figure 16 compares the pressure signatures for the SSGRID, SSG, MCAP and BG methods against the experimental fixed-transition results for a Gulfstream configuration at 1.7 body lengths below the aircraft for the on-track position. The CFD cases for this plot have been run assuming a fully turbulent condition. The feature in the experimental data at $\mathrm{X} / \mathrm{L}=2.4$ that none of the CFD methods picked up is a disturbance created by the first transition strip on the fuselage (see figure 11). Note that at some locations, such as the series of shocks and expansions at the nose (X/L near 2), the CFD data predicts stronger features than appear in the wind tunnel data. Discussions with wind tunnel engineers familiar with the data acquisition and reduction processes and uncertainty quantification suggest that the wind tunnel data features will likely reflect some reduction due to averaging, etc. Therefore, in assessing the "goodness" of a CFD result, higher magnitude peaks are generally considered more accurate even if they exceed the level of the wind tunnel data. The BG method generally captures more detail and retains larger peak magnitudes (positive and negative) than the other methods at this on-track location.

In figures 17, 18, and 19, the off-track pressure signatures (at off-track angles of 25, 35, and 53 degrees, respectively) for the SSG, MCAP, and BG methods have been compared with the experimental results for the fully turbulent flow condition. Pressure signatures have been extracted at $\mathrm{H} / \mathrm{L}$ of $1.73,1.756$, and 1.829 for the 25, 35, and 53 degrees cases, respectively. The BG method generally has higher peak magnitudes and more signature detail than the other two methods, though the SSG method is slightly better in the aft portion of the signature at higher off-track angles. The MCAP method in particular loses some of the pressure signature details at 53 degrees, especially in the nose region.

It was observed during the wind tunnel test that the flow over the wing was fully laminar and possibly over some portion of the fuselage. To compare with the experimental free-transition results, a new capability in the USM3D flow solver wherein the code can selectively turn off turbulence in the areas/locations of interest and treat those areas as laminar, has been used. In Figures 20, 21, 22, and 23 experimental free-transition results have been compared to that of CFD results computed with the wing treated as laminar and the remainder of the configuration treated as turbulent (designated as "mixed-flow"). In Figure 20, SSGRID, SSG, and BG pressure signatures have been compared for the on-track position. In Figures 21, 22, and 23 the pressure signatures of the SSG and BG methods are compared with the experimental results for the off-track angles of 25, 35, and 53 degrees. Again, the BG method seems to do slightly better than the other two methods, with the exception of SSGRID giving better match to the expansion peak at $\mathrm{X} / \mathrm{L}=2.8$ for the on-track signature (as shown in figure 20). As can be seen from figures 22 and 23, SSG seems to do better in aft portion. Since none of the methods fully captured the large 
expansion at $\mathrm{X} / \mathrm{L}=2.9$ that is also apparent in the off-track signatures, it was decided to run a fully-laminar case and see how it compares with the experimental free-transition case. Figure 24 shows a comparison of three modes of SSG results (fully turbulent, mixed-flow, and fully laminar) with both experimental free-transition and fixedtransition results for the on-track position. As can be seen the laminar case does a better job for the expansion part but not as good for the compression part $(\mathrm{X} / \mathrm{L}=2.6)$ compared with the mixed-flow and turbulent cases. Figure 25 shows a comparison of three modes of BG results (fully-turbulent, mixed-flow, and fully-laminar) with both experimental free-transition and fixed-transition results for the on-track position. The results are similar to those in figure 24, with the laminar case doing a better job for the expansion part but not as good for the compression part compared with the transitional case.

\section{Lockheed Martin (LM) Configuration}

Lockheed Martin's configuration is a novel concept with extensive nose droop for the forward pressure signature shaping, and they have developed strategies for aft shaping under a current NASA Research Announcement (NRA) award $^{10}$. The Supersonics Project under NASA's Fundamental Aeronautics Program is working toward developing technologies to enable future civilian supersonic aircraft to fly efficiently, with low noise, reduced emissions, and without restricted flight corridors. The aircraft is designed to be 230 feet long and it appears to have extensive shaping with tailored wing surfaces for low sonic boom and drag. Two conventionally mounted nacelles and a third nacelle integrated into the V-tail empennage will be used to power the vehicle. A three nacelle arrangement likely will provide reduced sonic boom compared with a conventional four nacelle arrangement.

The grid generation sequence on the LM configuration is shown in Figure 26. Figures 26a and 26b show the sourcing in Gridtool in the side and front views respectively. Figure 26c shows the surface grid. The inner core grid size without the collar is 21 million cells. Figure 26d shows the symmetry plane grid after adding the collar and stretching using the BG method. The final grid is approximately 29 million cells. Pressure signatures have been obtained for both on-track and off-track angle of 40 degrees conditions. It was also proposed to investigate if one single grid would suffice for both conditions. As the experimental data for the on-track and off-track conditions were obtained at different angles of attack (2.6 and 3.0 degrees, respectively), it is not possible to directly evaluate the use of a single grid. Instead, the effect of grid clustering will be evaluated separately at each condition. Using BG methodology, two grids were generated for each of the angles of attack of 2.6 and 3.0 degrees, one with on-track grid clustering and the other with clustering near the off-track angle of 40 degrees. Figure 27 shows the on-track pressure signatures obtained from the two grids generated for angle of attack of 2.6 degrees, compared with the experimental results. The flow conditions are a Mach number of 1.6, angle of attack of 2.6 degrees, and Reynolds number of 8.0 million based on a reference length of 22.365 inches. Pressure signatures were extracted at 0.94 body lengths below the aircraft model. As can be seen both grids yield similar signatures. Figure 28 shows the comparison of the 40 degree off-track pressure signatures obtained from the two grids generated for an angle of attack of 3.0 degrees, compared with that of the experiment. The off-track grid clustering results are in good agreement with the experiment except that the CFD results seem to give a higher compression peak. The on-track grid clustering does not capture the compression and expansion features at the nose as well as the off-track grid clustering. The results from figures 27 and 28 seem to indicate that a single grid with clustering near the maximum off-track angle might be acceptable for analysis and/or design across a spectrum of track angles.

\section{Straight Line Segmented Leading Edge (SLSLE) Model}

The SLSLE model is a wing-body configuration with a straight-line segmented leading edge wing. The model leading edge consists of 3 line segments with leading edge breaks at 13.3 and 23.1-percent span. A sonic boom wind tunnel test was conducted on the model in the NASA Langley 4- by 4- Foot Unitary Plan Wind Tunnel (UPWT) in 2011. The purpose of the test was to determine whether accurate sonic boom measurements could be obtained while continuously moving the SLSLE model past a conical pressure probe ${ }^{18}$. An earlier wind tunnel test was also conducted at NASA GRC 10- by 10-ft Supersonic wind tunnel in May 2005, wherein pressure signatures at larger distances below the model could be extracted. The small size of the wind tunnel model allowed boom signatures to be obtained up to 10 body lengths below the model at Mach 2.0.

The grid generation sequence on the SLSLE configuration is shown in Figure 29. Figures 29a and 29b show the sourcing in Gridtool in the side and front views, respectively. Figure 29c shows the surface grid. The inner core grid 
size (for an overall source factor of 2.0 in AUTOSRC) without the collar is 23.9 million cells. Figure 29d shows the symmetry plane grid after adding the collar and stretching using the BG method. The final grid is approximately 64.7 million cells.

A grid sensitivity study has been conducted on the SLSLE configuration to assess the initial core grid size needed to accurately predict the pressure signature. This is accomplished through varying the overall source factor in the AUTOSRC code. Three overall source factors have been studied, namely, 1.0, 1.5 and 2.0. The grid becomes coarser with an increase in the source factor, with the primary impact being the grid spacing on the surface of the configuration. For this study, sourcing on the boom cylinder has been kept the same and only the line sources on the configuration have been varied. The far-field boundary of the grid has been stretched up to 3 body lengths. The grid sizes and source factors are listed in Table 1.

Table 1. Grid Parameters

\begin{tabular}{|c|c|c|}
\hline $\begin{array}{c}\text { Overall Source } \\
\text { Factor }\end{array}$ & $\begin{array}{c}\text { Inner Core Grid Size } \\
\text { (number of cells) }\end{array}$ & $\begin{array}{c}\text { Final Grid Size after Adding } \\
\text { the Collar } \\
\text { (number of cells) }\end{array}$ \\
\hline 2.0 & $23,911,939$ & $64,687,366$ \\
\hline 1.5 & $25,799,139$ & $66,792,168$ \\
\hline 1.0 & $29,366,007$ & $68,768,502$ \\
\hline
\end{tabular}

Figure 30 shows the comparison of the on-track pressure signatures for the three source factors 1.0, 1.5, and 2.0 with that of the experiment. The flow conditions are a Mach number of 2.0, angle of attack of 2.3 degrees and Reynolds number of 1.5 million based on a reference length of 9.0 inches. As can be seen, the results for all three source factors are essentially identical and compare very well with the experimental results at 1.5 body lengths below the model.

A study was also conducted wherein the S2 factor (maximum spacing between the layers) in the BG code was varied to optimize the grid size. In this study, the initial spacing factor (S1) and the rate of increase (R1) were each held constant at a value of 2.0, and the outer boundary was stretched to three body lengths. While a larger S2 factor will allow a larger signature distance to be reached using fewer cells, there was concern that the larger cell aspect ratios may lead to code convergence issues. Two S2 factors were studied, namely, 50 and 100. Increasing S2 from 50 to 100 for a case where the overall source factor in AUTOSRC is 1.0, reduces the grid size from 68.8 million cells to 53.2 million cells. Figure 31 shows the comparison of the pressure signatures of S2 of 50 and 100 with that of the experiment at 1.5 body lengths below the model. No significant deterioration in the pressure signature is noticed by doubling S2 at this distance. Figure 32 shows the comparison of CFD and experimental results at 10 body lengths below the aircraft model. For this case also, grids have been generated for S2 of 50 and 100. Increasing S2 from 50 to 100 reduces the grid size from 101.6 million to 64.3 million cells. No significant deterioration in pressure signature was noticed due to increase in S2.

The grid modification process with BG (adding collar and grid stretching and shearing) is extremely fast. For example, the 101 million cell final grid for the SLSLE case above could be generated in less than a minute starting from a 21.9 million cell inner grid. In all of the grid generation cases shown in this paper, cells with negative volumes were not an issue and they cleaned up in very few cycles (typically less than 5). BG method also gave better convergence compared to SSGRID or SSG method. With the BG method, obtaining the pressure signatures at 10 body lengths below the model was not an issue, which could not be accomplished with the MCAP method. 


\section{Conclusions}

Two new grid modification codes, Stretched and Sheared Grid - Modified (SSG) and Boom Grid (BG), have been developed for low boom analysis. SSG was tested on a Gulfstream configuration and BG was evaluated on Gulfstream, Lockheed Martin and SLSLE configurations. The codes have been tested for both on-track and off-track simulations up to 53 degrees. Good agreement has been obtained between the CFD simulations and the wind tunnel experimental results. Grid sensitivity studies on the SLSLE model indicate that using an overall source factor of 2.0 in AUTOSRC is sufficient to capture the pressure signatures correctly. Increasing the maximum spacing factor in BG code from 50 to 100 will decrease the final grid size by about $40 \%$ without any significant degradation of the solution accuracy. The BG methodology was found to be extremely robust and fast. Large grids of about 100 million cells could be generated in less than a minute and negative volume cells cleaned up in less than 5 cycles. The BG method also gave better convergence compared to SSGRID or SSG method. With the BG method, pressure signatures at 10 body lengths below the model could be obtained, which could not be accomplished with the MCAP method. The off-track clustering capability in BG allowed accurate signatures to be obtained for off-track angles of up to 53 degrees. Initial indications are that a single grid clustered at a maximum off-track angle of 40 degrees may be sufficient for both on- and off-track signatures.

\section{Acknowledgments}

The work done was sponsored by NASA Fundamental Aeronautics Program Supersonics Project under contract NNL09AM01T. The version of the USM3D code with the mixed-flow capability was provided by Dr. Mohagna Pandya of NASA Langley Research Center.

\section{References}

${ }^{1}$ CRISP CFD User’s Manual, Version 1.5,” Combustion Research and Flow Technology, Inc., 6210 Keller's Church Road, Pipersville, PA 18947, February 2007.

${ }^{2}$ Frink,N. T.; Pirzadeh, S.Z.; Parikh, P.C.; Pandya, M.J.; and Bhat, M.K., "The NASA Tetrahedral Unstructured Software System”, The Aeronautical Journal, Vol. 104, No. 1040, October 2000, pp. 491-499.

${ }^{3}$ Samareh, Jamshid, “GridTool: A Surface Modeling and Grid Generation Tool,” Proceedings of the Workshop on Surface Modeling, Grid Generation and Related Issues in CFD Solutions, NASA CP-3291, May 9-11, 1995.

${ }^{4}$ Campbell, Richard L.; Carter, Melissa B.; Deere, Karen A.; and Waithe, Kenrick A., "Efficient Unstructured Grid Adaptation Methods for Sonic Boom Prediction.” AIAA 2008-7327, August 2008

${ }^{5}$ Pirzadeh S., "Three-Dimensional Unstructured Viscous Grids by the Advancing Layers Method.” AIAA Journal, Vol. 34, No. 1, January 1996, pp. 43-49

${ }^{6}$ Lohner R and Parikh P., “Three-Dimensional Grid Generation by the Advancing Front Method.” International Journal for Numerical Methods in Fluids Volume 8, pp 1135-1149 (1988)..

${ }^{7}$ Pirzadeh S., “Advanced Unstructured Grid Generation for Complex Aerodynamics Applications.” AIAA-20087178, August 2008.

${ }^{8}$ Li, Wu; Campbell, Richard; Geiselhart, Karl; Shields, Elwood; Nayani, Sudheer; Shenoy, Rajiv, "Integration of Engine, Plume, and CFD Analyses in Conceptual Design of Low-Boom Supersonic Aircraft.” AIAA 2009-1171, January 2009.

${ }^{9}$ Park, Michael A., "Low Boom Configuration Analysis with FUN3D Adjoint Simulation Framework”, AIAA2011-3337, 48th AIAA Aerospace Sciences Meeting Including the New Horizons Forum and Aerospace Exposition, 4 - 7 January 2010, Orlando, Florida

${ }^{10}$ Cliff, Susan E., Elmiligui, Alaa A., Campbell, Richard L., Thomas, Scott D., "Evaluation of Refined Tetrahedral Meshes with Projected, Stretched, and Sheared Prism Layers for Sonic Boom Analysis”, $29^{\text {th }}$ AIAA Applied Aerodynamics Conference, AIAA 2011-3338, June 2011, Honolulu, Hawaii. 
${ }^{11}$ Jameson, A., and Baker, T., "Improvements to the Aircraft Euler Method,” AIAA Paper-87-0452, 1987.

${ }^{12}$ Hooker, J.R., "Transonic Efficient Mobility Planform Optimization (TEMPO) - Lockheed Martin - Volume 1 Program Final Report,” AFRL-RB-WP-TR-2008-3121, Wright Patterson, OH 2008.

${ }^{13}$ Frink, N. T., "Three-Dimensional Upwind Scheme for Solving the Euler Equations on Unstructured Tetrahedral Grids”, Ph. D. Dissertation, Virginia Polytechnic Institute and State University, September 1991.

${ }^{14}$ Spalart, P.; and Allmaras, S.A., “One-Equation Turbulence Model for Aerodynamic Flows.” AIAA 92-0439, January 1992.

${ }^{15}$ Menter, F.R., “Improved Two-Equation k-omega Turbulence Models for Aerodynamic Flows.” NASA TM103975, October 1992.

${ }^{16}$ Pandya, Mohagna J.; Abdol-Hamid, Khaled S.; Campbell, Richard L.; and Frink, Neal T.: Implementation of Flow Tripping Capability in the USM3D Unstructured Flow Solver. AIAA-2006-0919, January 2006.

${ }^{17}$ Campbell, Richard L.; Campbell, Matthew L.; and Streit, Thomas: "Progress Toward Efficient Laminar Flow Analysis and Design.” AIAA-2011-3527, June 2011.

${ }^{18}$ Elmiligui,A., Wilcox, F., Cliff, S.,Thomas, S., "Numerical Prediction of Sonic Boom Signatures for a Straight Line Segmented Leading Edge Model.”, Seventh International Conference on Computational Fluid Dynamics (ICCFD7), Big Island, Hawaii, July 9-13, 2012. 


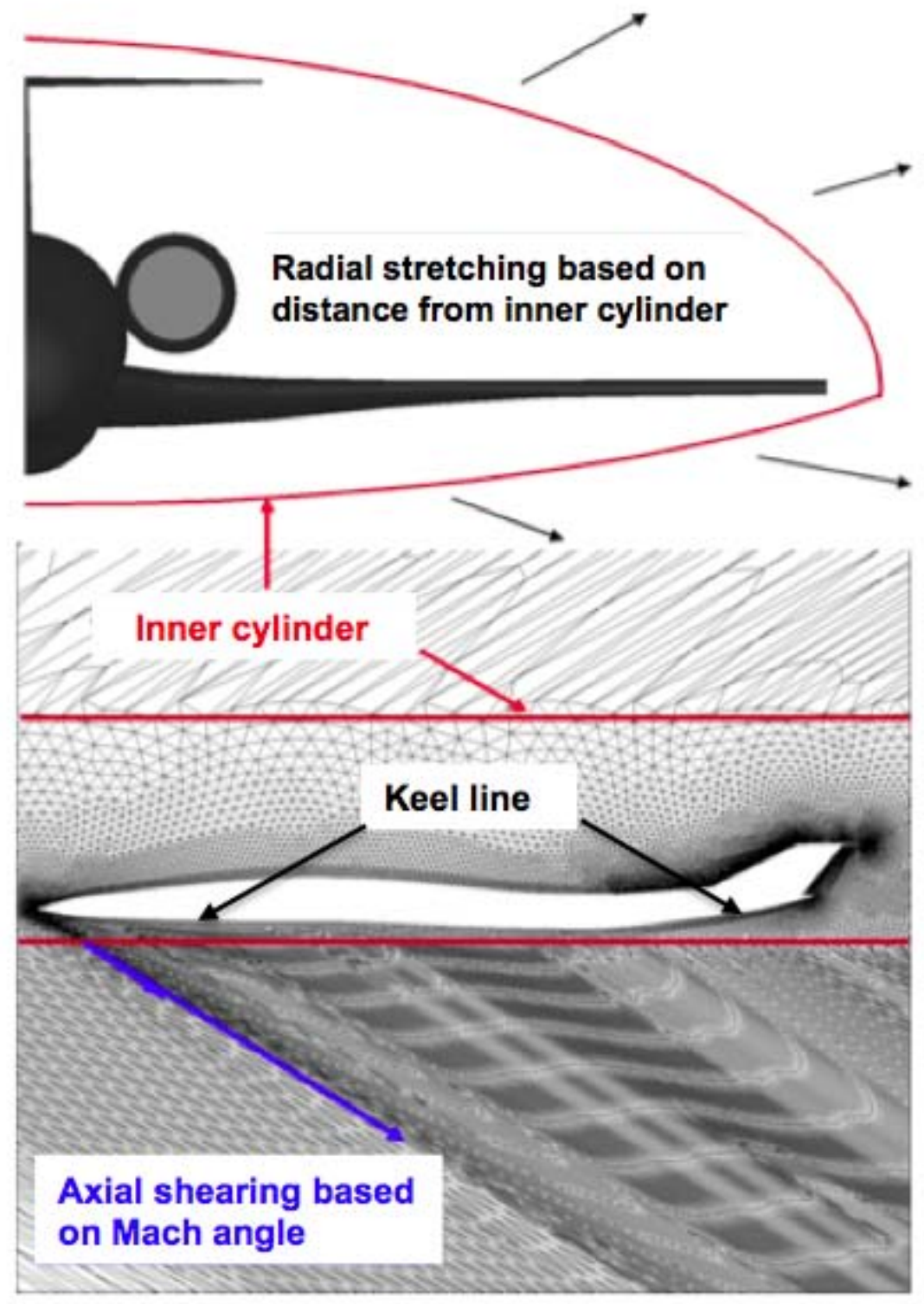

Figure 1. Grid stretching and shearing controlled by an inner cylinder. 


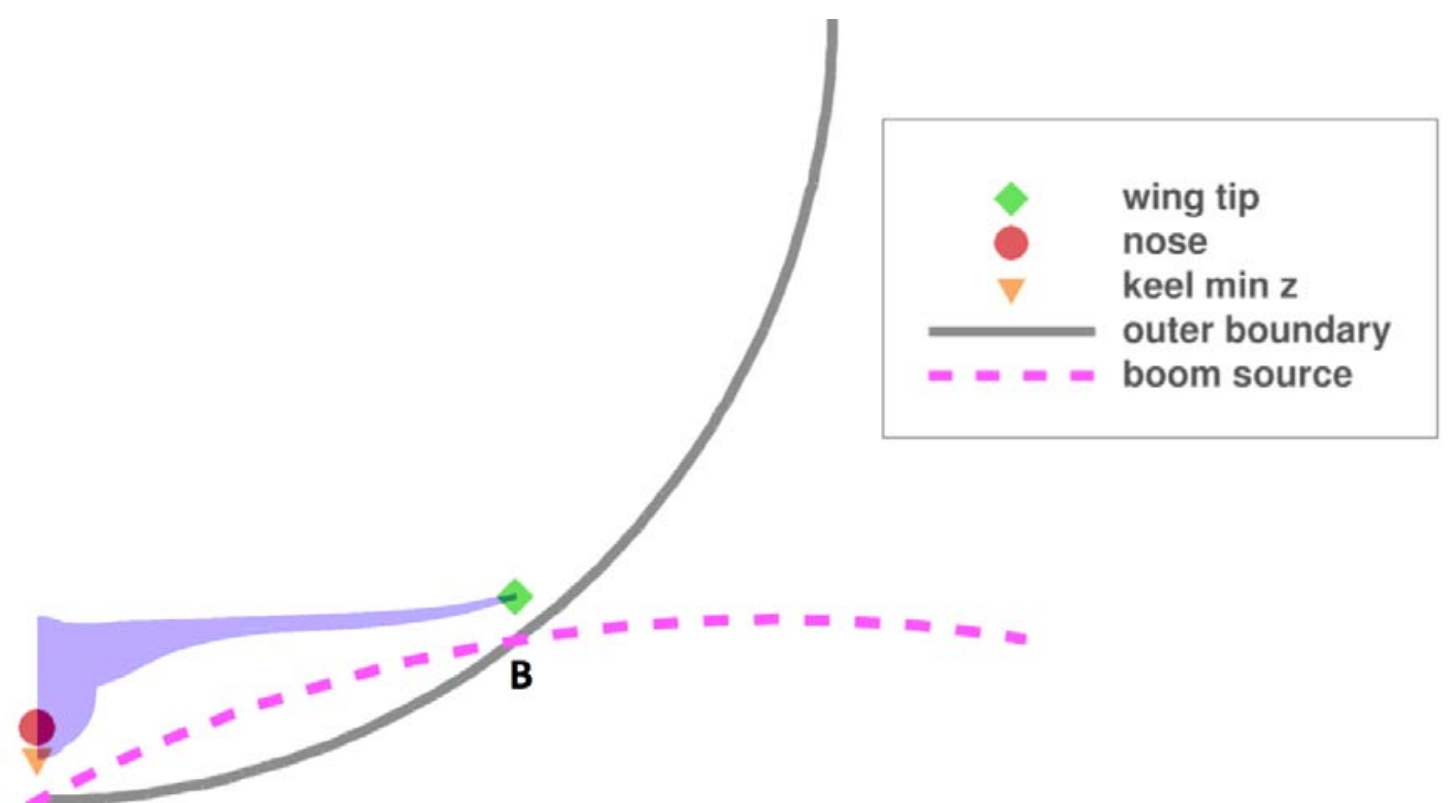

A

Figure 2. Location of the cylindrical boom source.

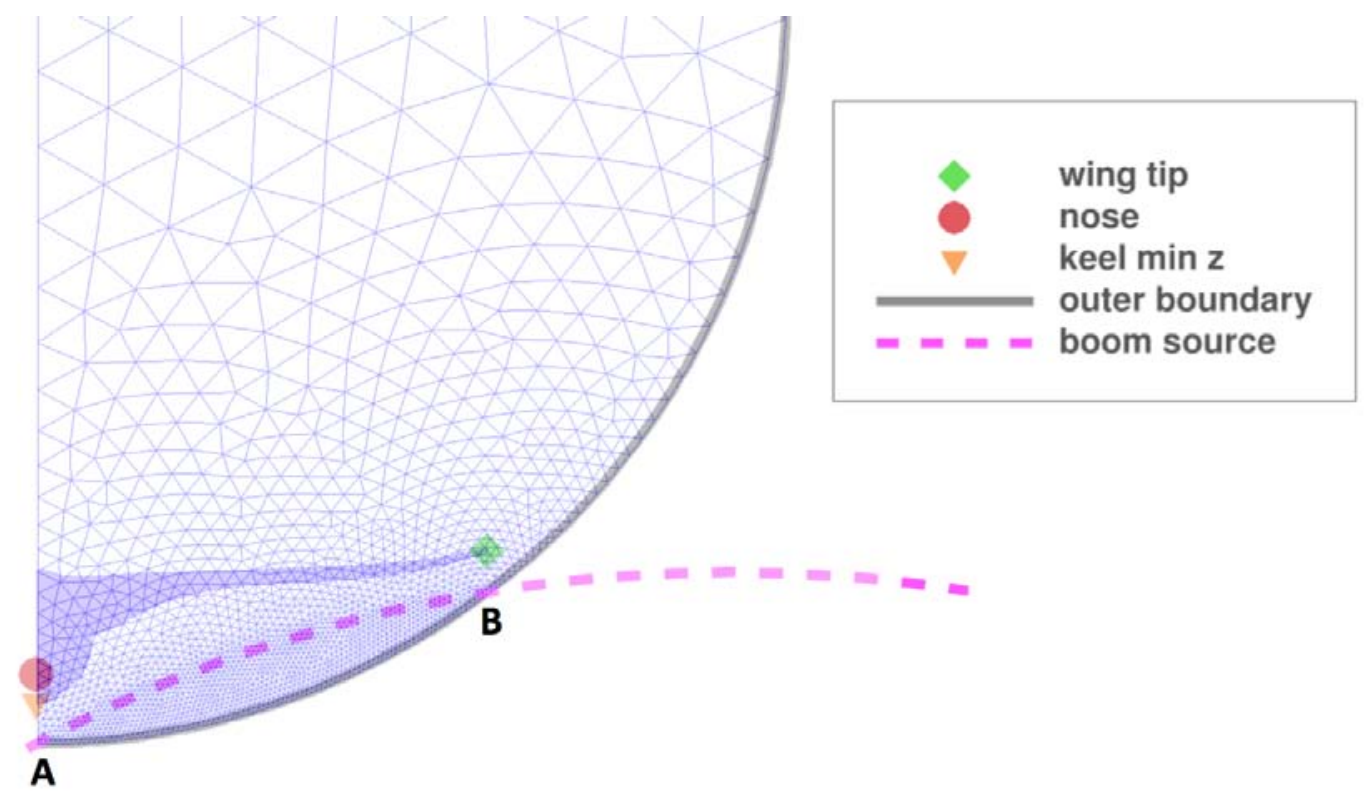

Figure 3. Core grid inflow plane. 


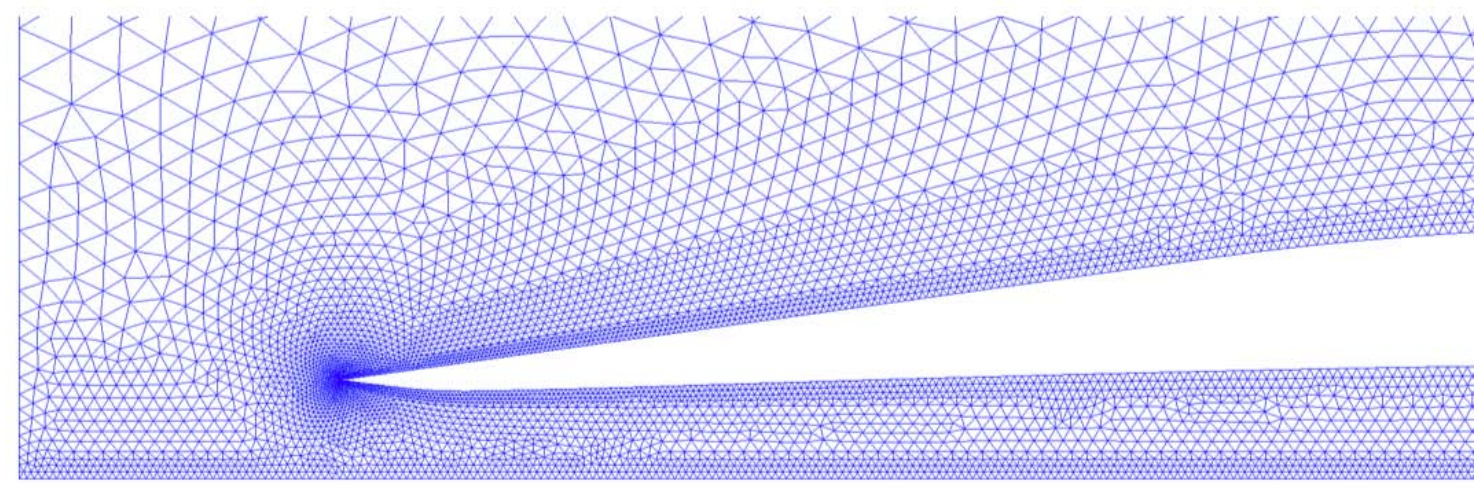

Figure 4. Symmetry plane in the nose region.

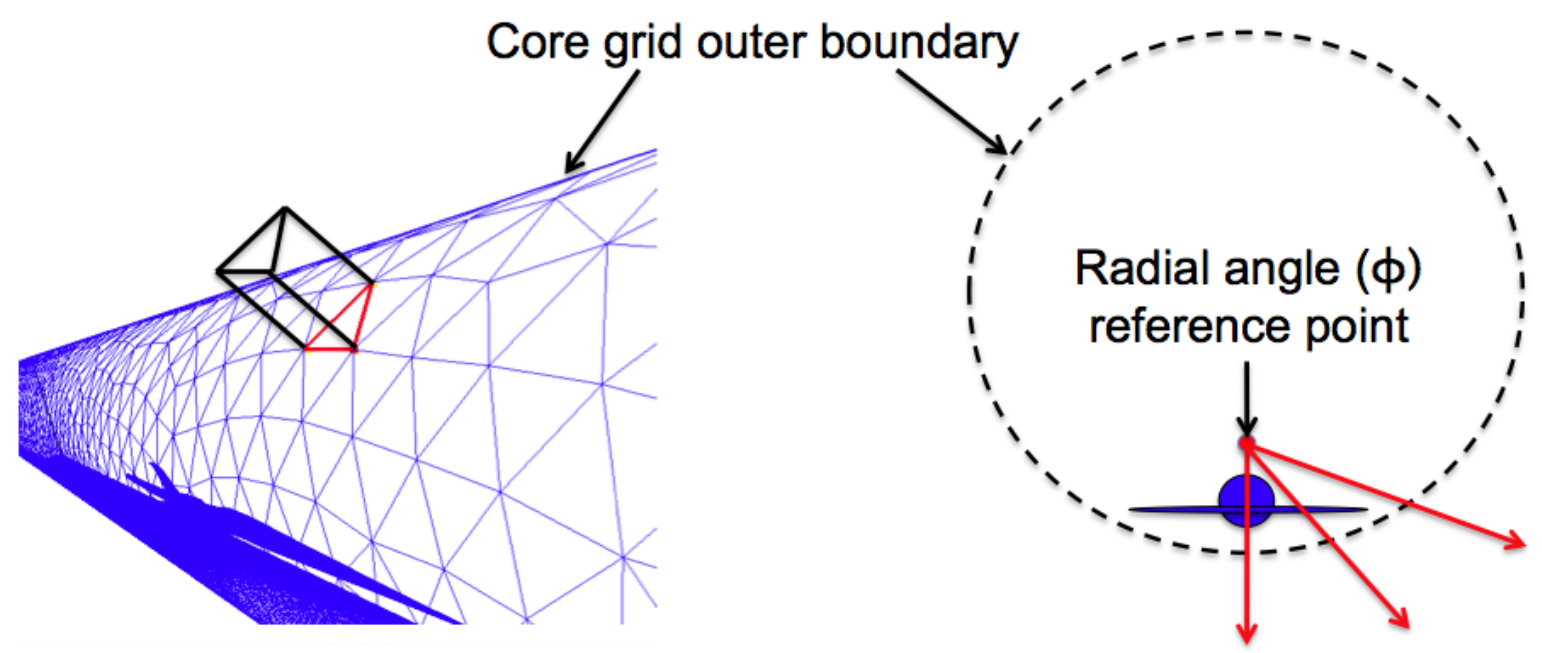

Figure 5. Prism extrusion process in the BG grid methodology. 


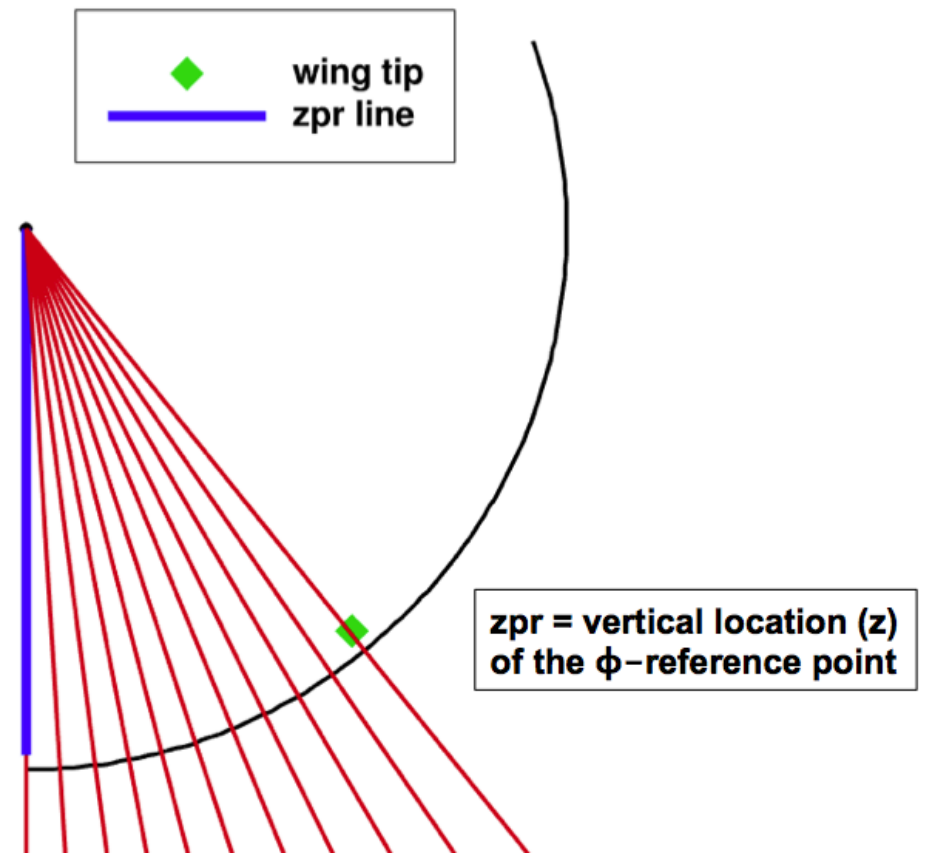

Figure 6. Zpr line for the on-track signature case.

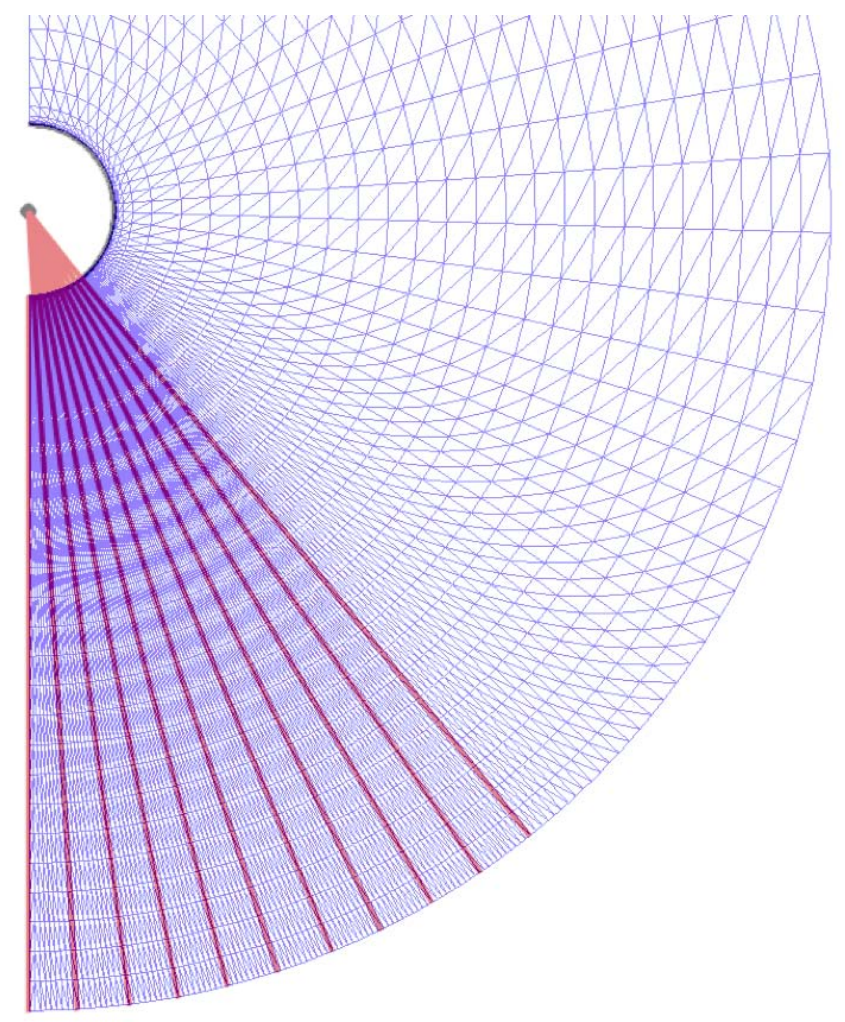

Figure 7. Resulting inflow plane for the new outer grid. 


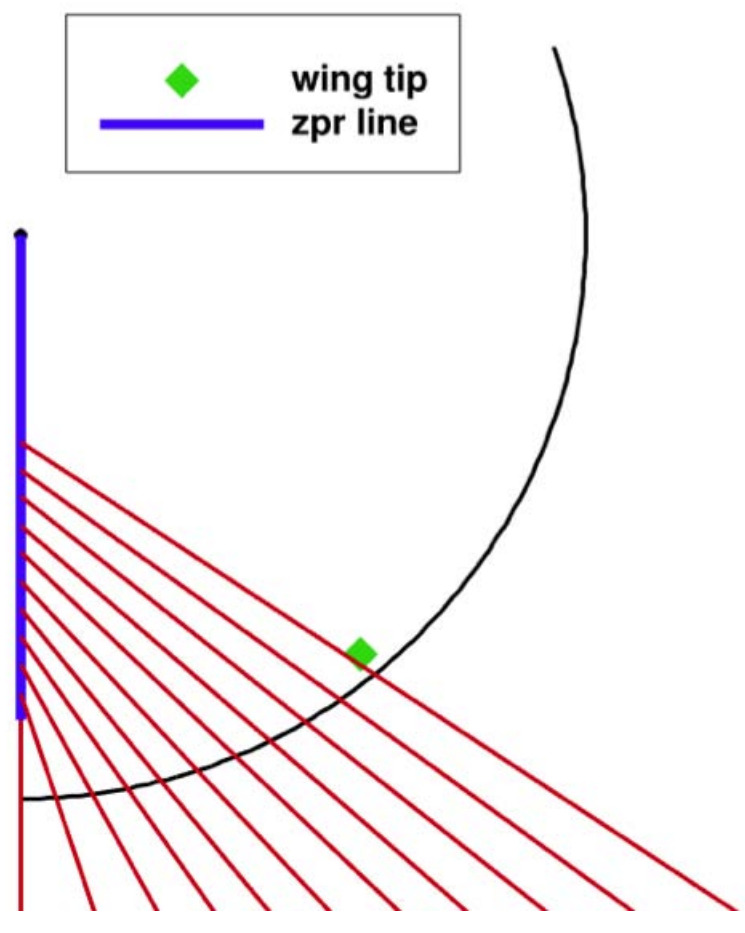

Figure 8. Zpr line location for the off-track signature case.

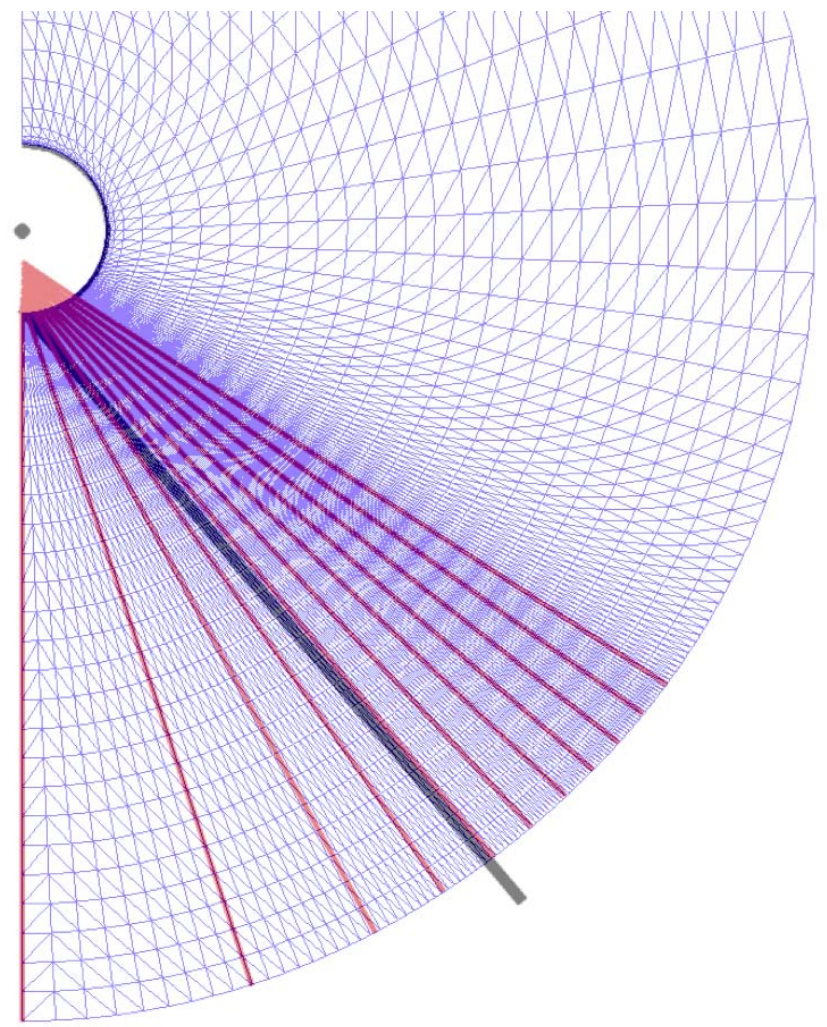

Figure 9. Grid clustering for the desired off-track angle. 


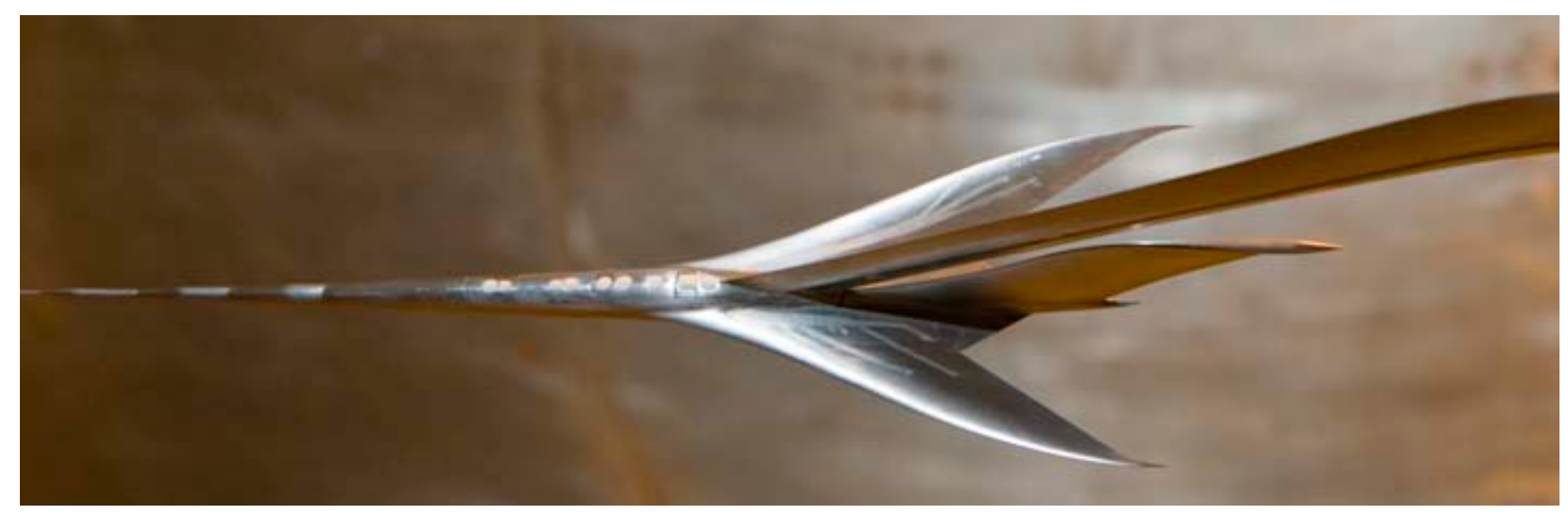

Figure 10. Experimental Gulfstream boom model.

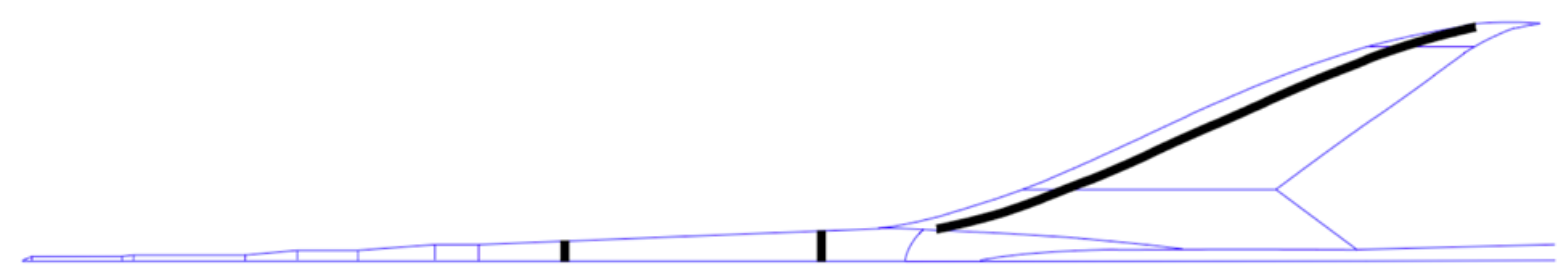

Figure 11. Schematic of trip locations on the model (denoted in black). 

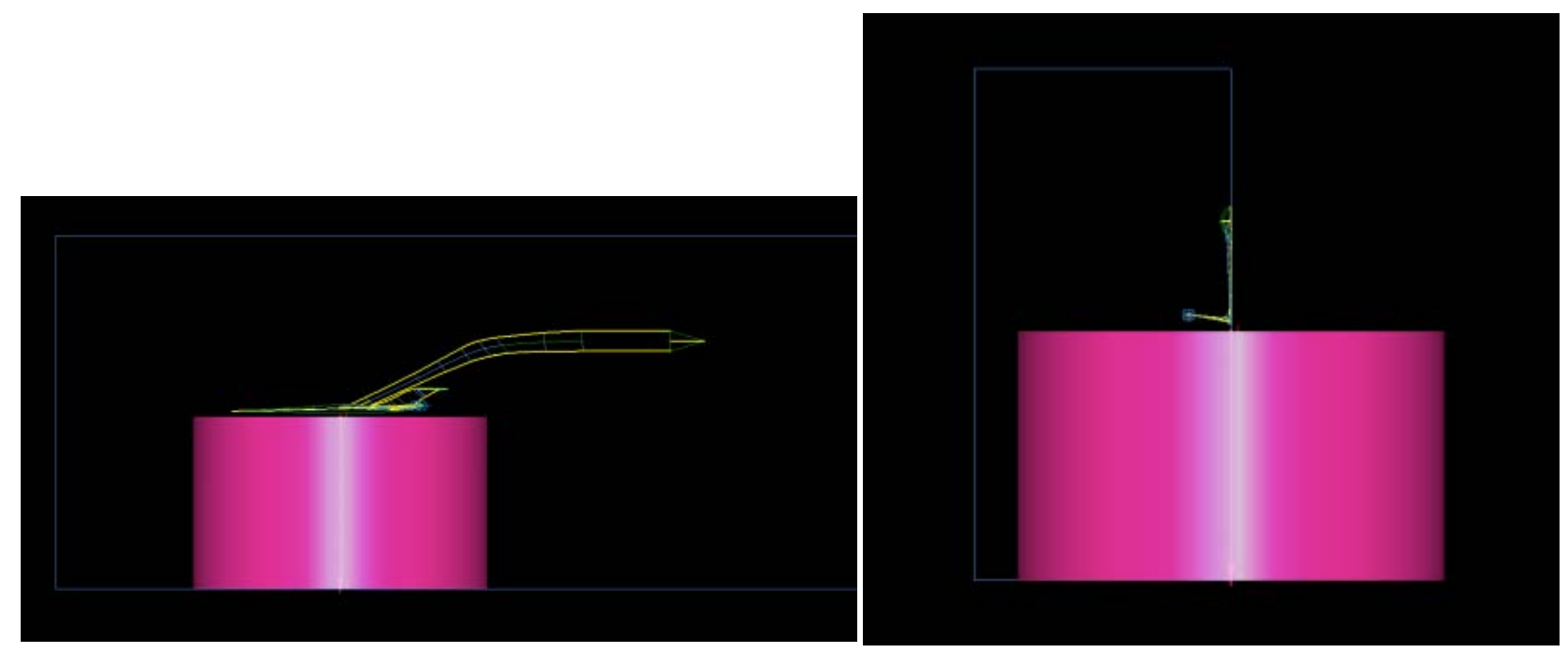

a. Sourcing in GridTool - side view

b. Sourcing in GridTool - rear view
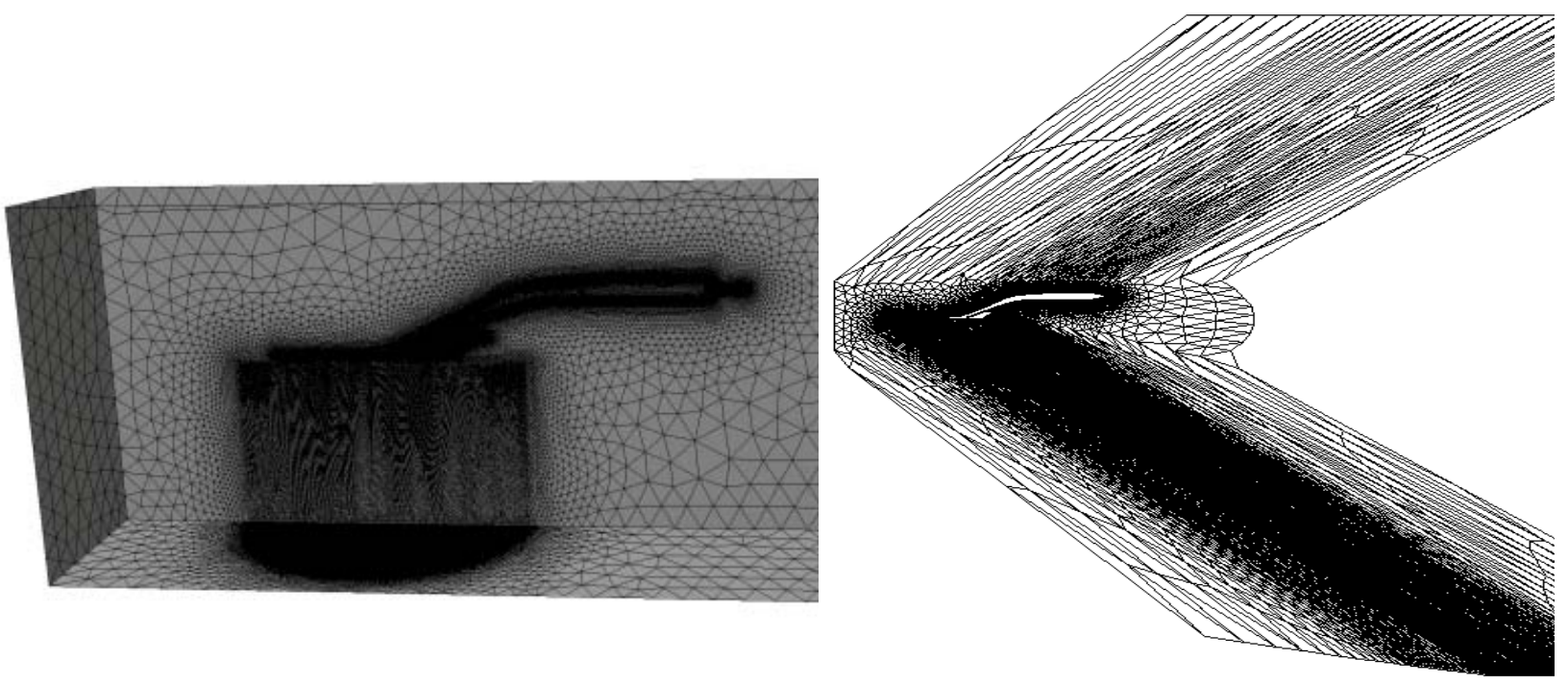

c. Surface grid from VGRID

d. Symmetry plane grid after stretching

Figure 12. Grid generation for the SSGRID method - Gulfstream configuration. 


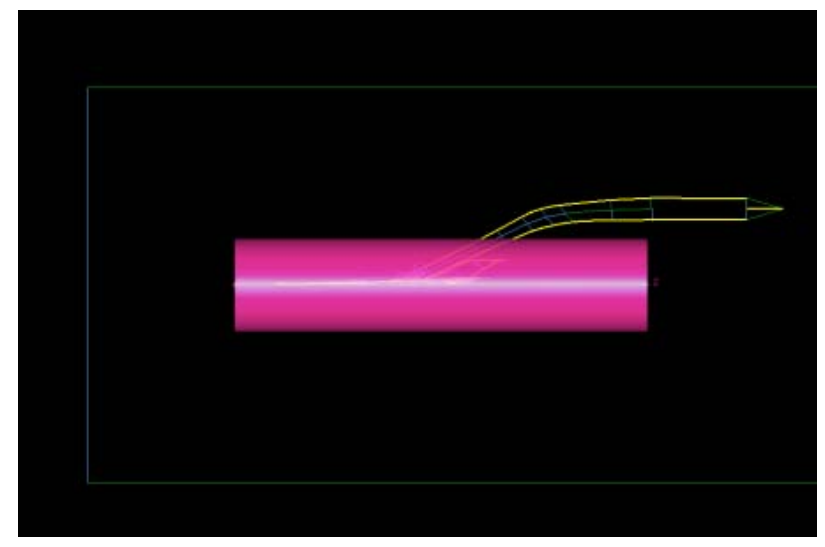

a. Sourcing in GridTool - side view

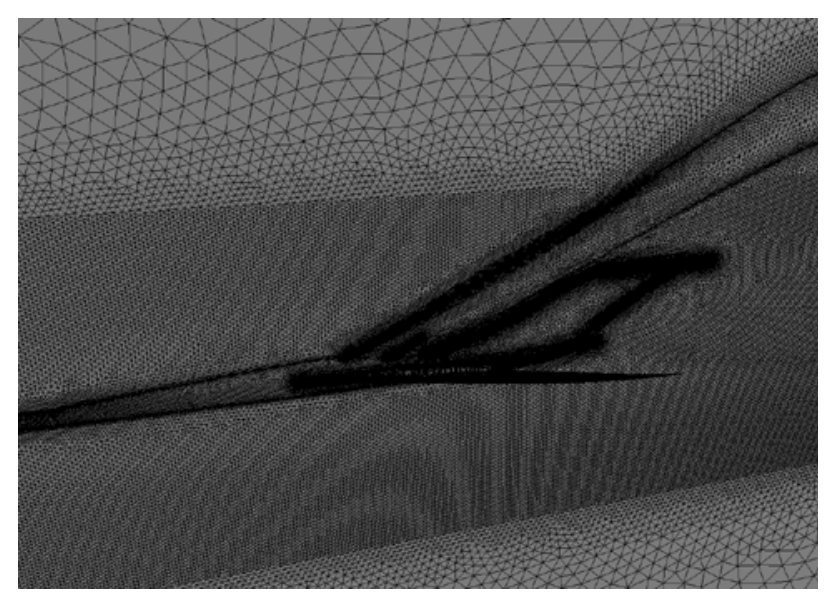

c. Surface grid from VGRID

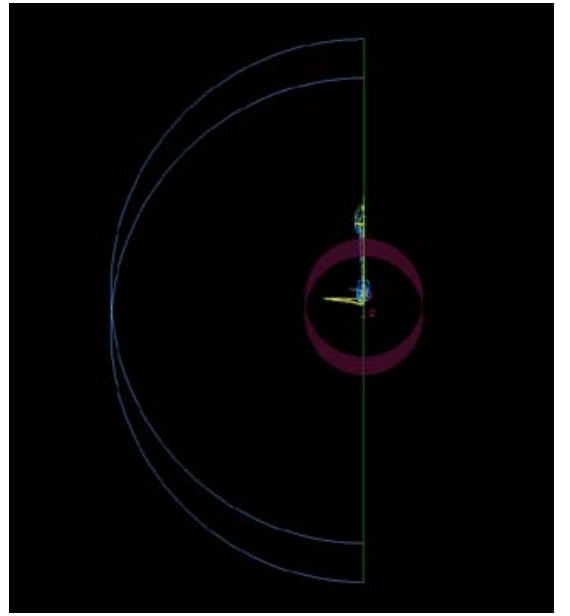

b. Sourcing in GridTool - rear view (slightly tilted to show boom source)

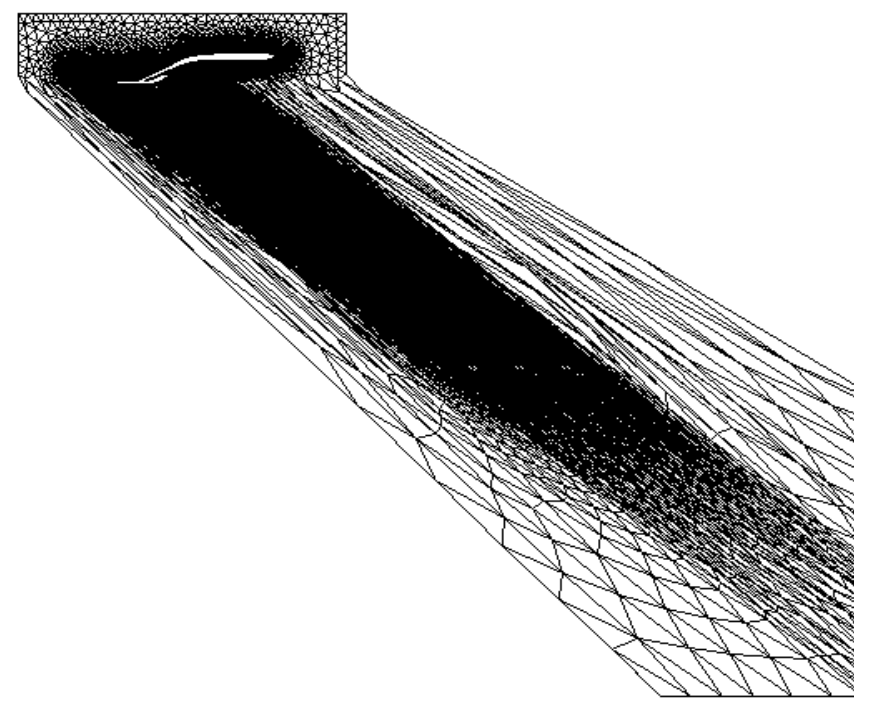

d. Symmetry plane grid after stretching

Figure 13. Grid generation for the SSG method - Gulfstream configuration. 

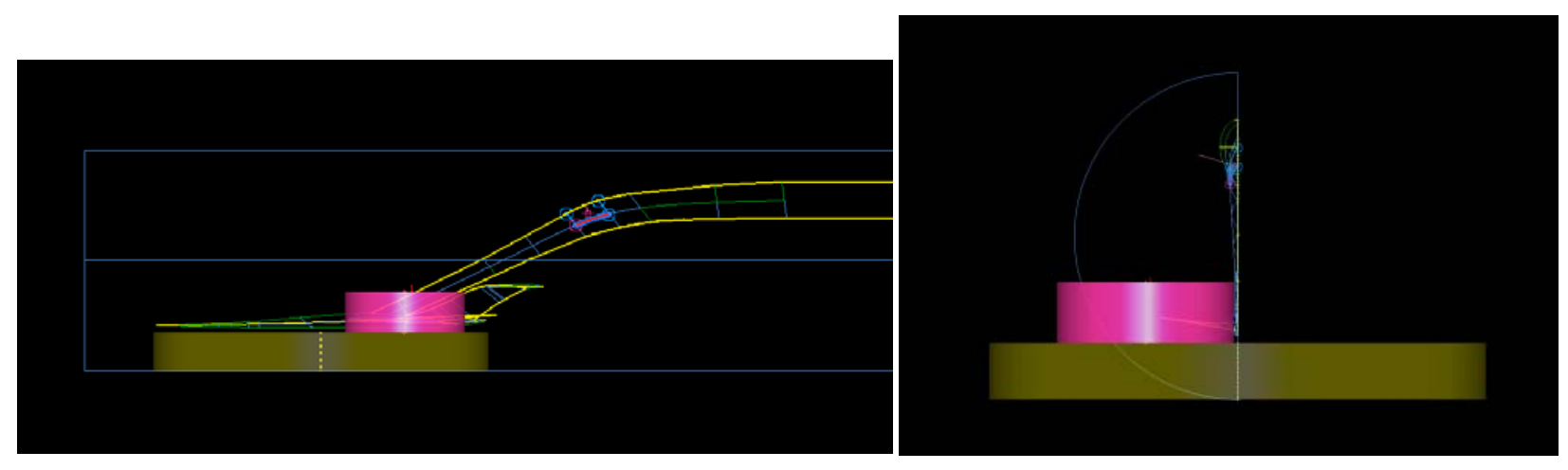

a. Sourcing in GridTool - side view

b. Sourcing in GridTool - rear view

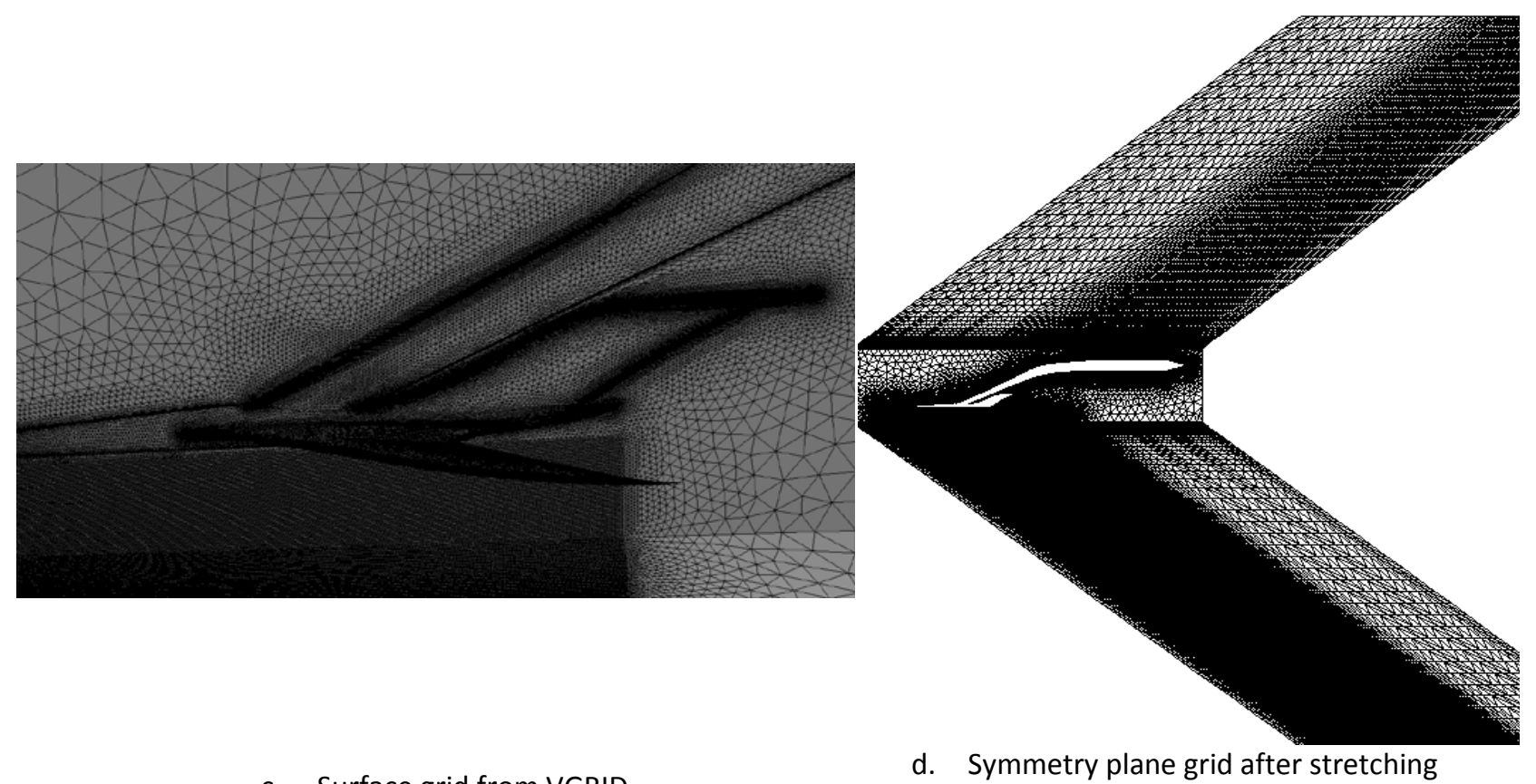

c. Surface grid from VGRID

d. Symmetry plane grid after stretching

Figure 14. Grid generation for the MCAP method - Gulfstream configuration. 


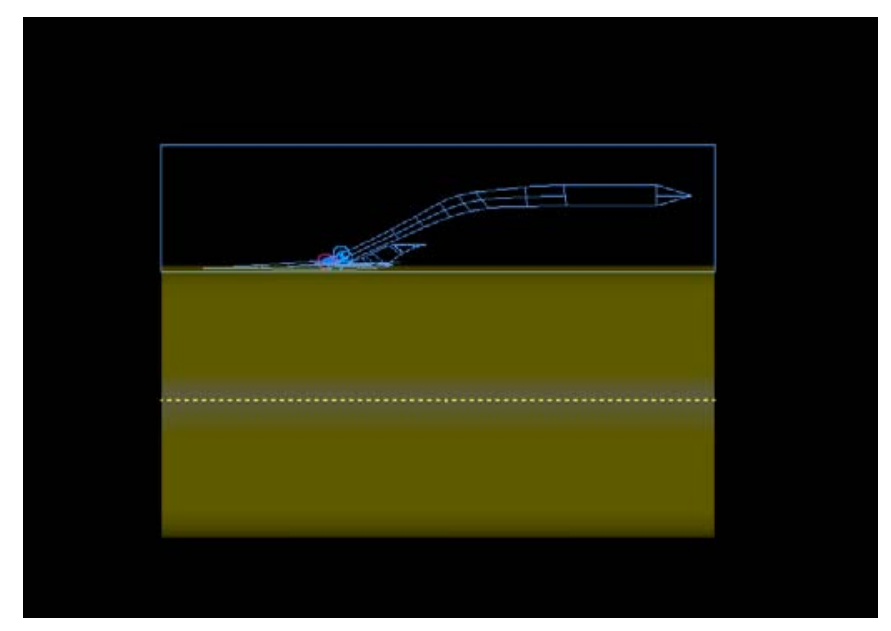

a. Sourcing in GridTool - side view

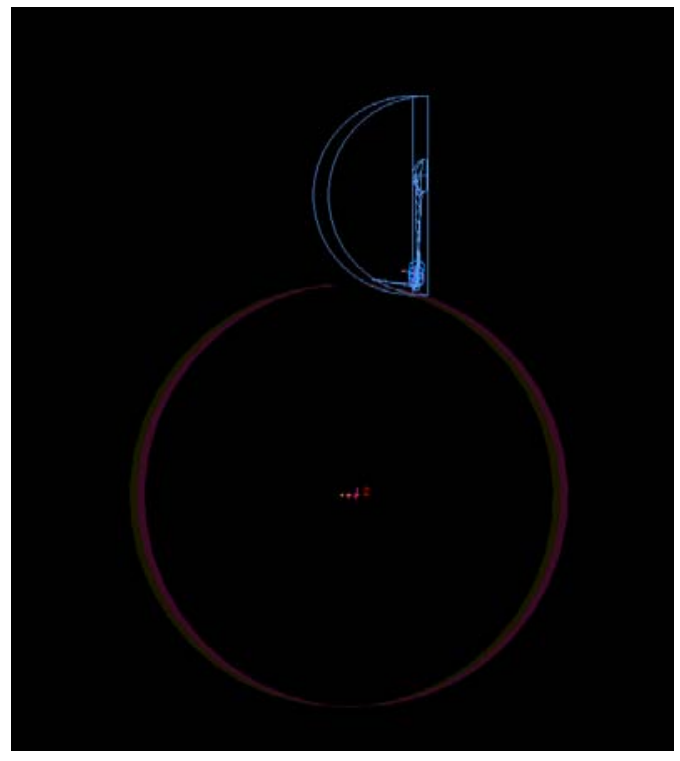

b. Sourcing in GridTool - rear view (slightly tilted to show volume source)

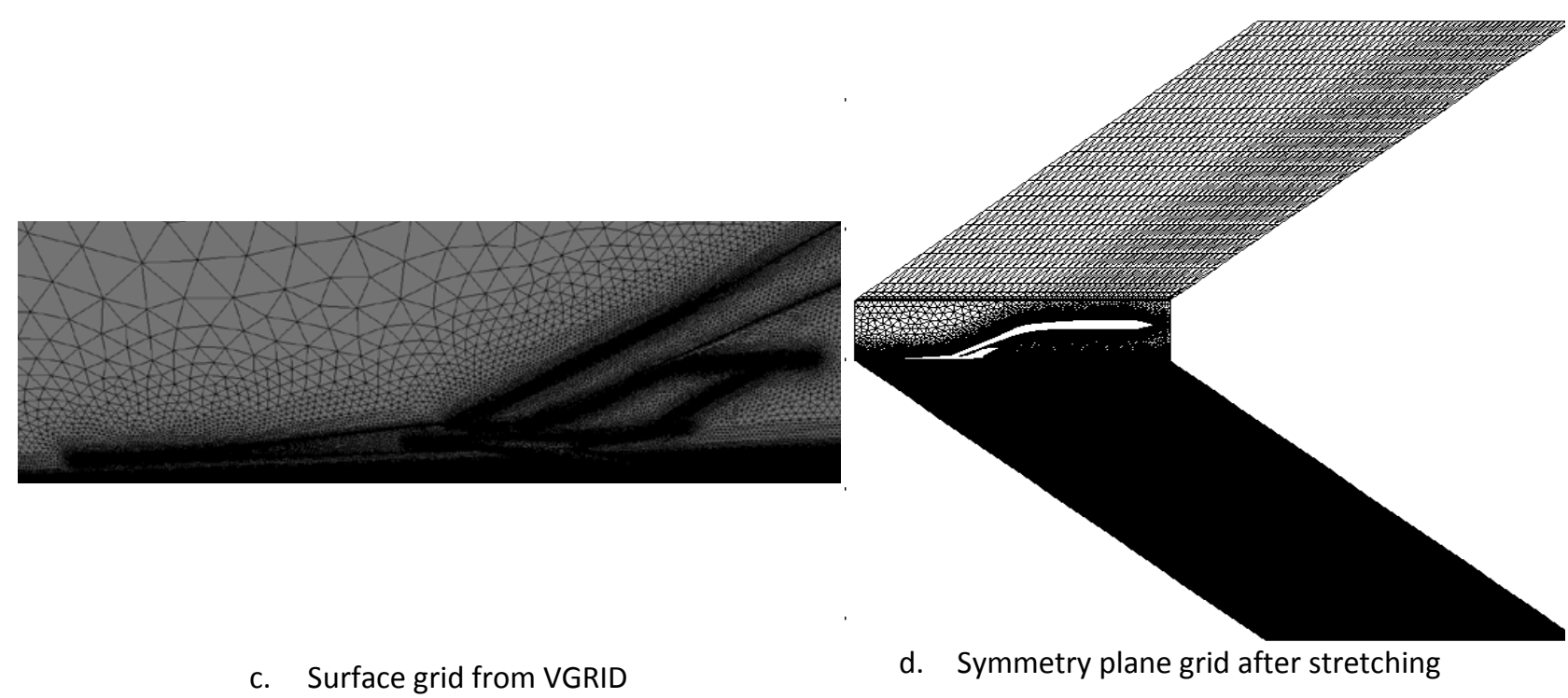

Figure 15. Grid generation for the BG method - Gulfstream configuration. 


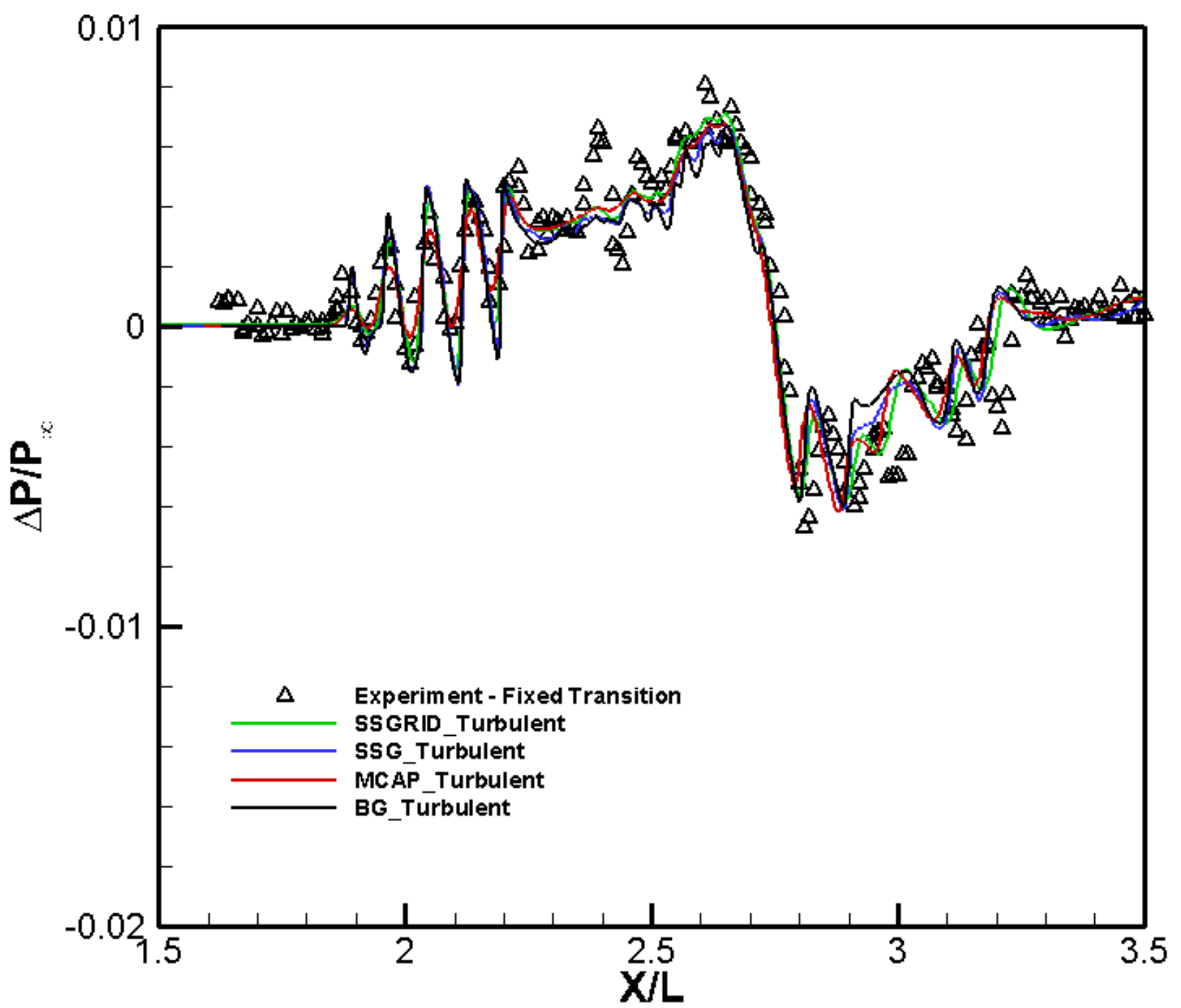

Figure 16. Comparison of experimental data with on-track pressure signatures of grid adaption schemes SSGRID, SSG, MCAP, and BG for turbulent flow condition on the Gulfstream configuration $(H / L=1.7$, Mach number $=1.56, \alpha=0.256$ degrees, $\operatorname{Re}_{\mathrm{L}}=3.86$ million). 


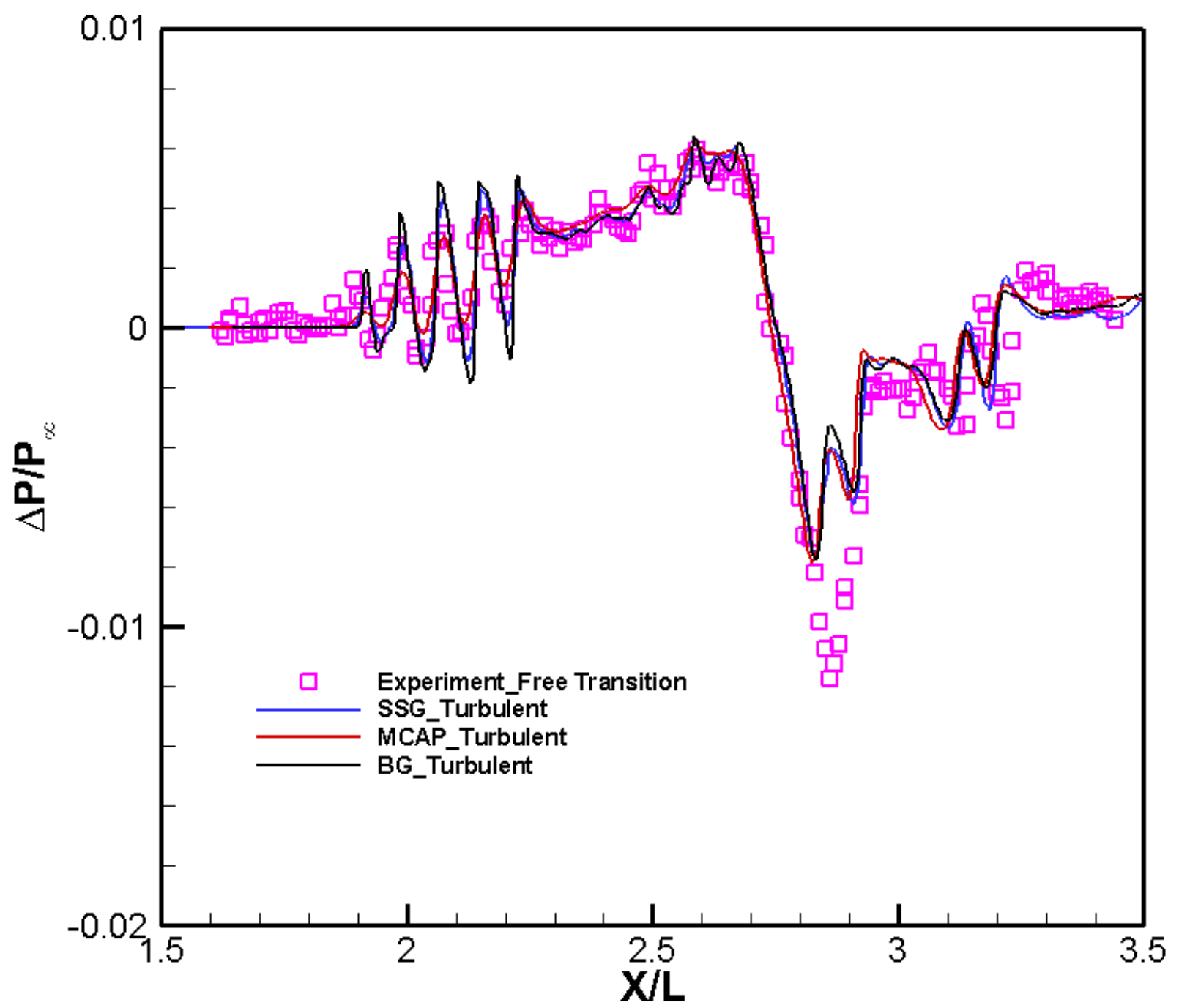

Figure 17. Comparison of experimental data with 25 degrees off-track pressure signatures of grid adaption schemes SSG, MCAP, and BG for turbulent flow condition on the Gulfstream configuration $(H / L=1.73$, Mach number=1.56, $\alpha=0.256$ degrees, $\operatorname{Re}_{\mathrm{L}}=3.86$ million). 


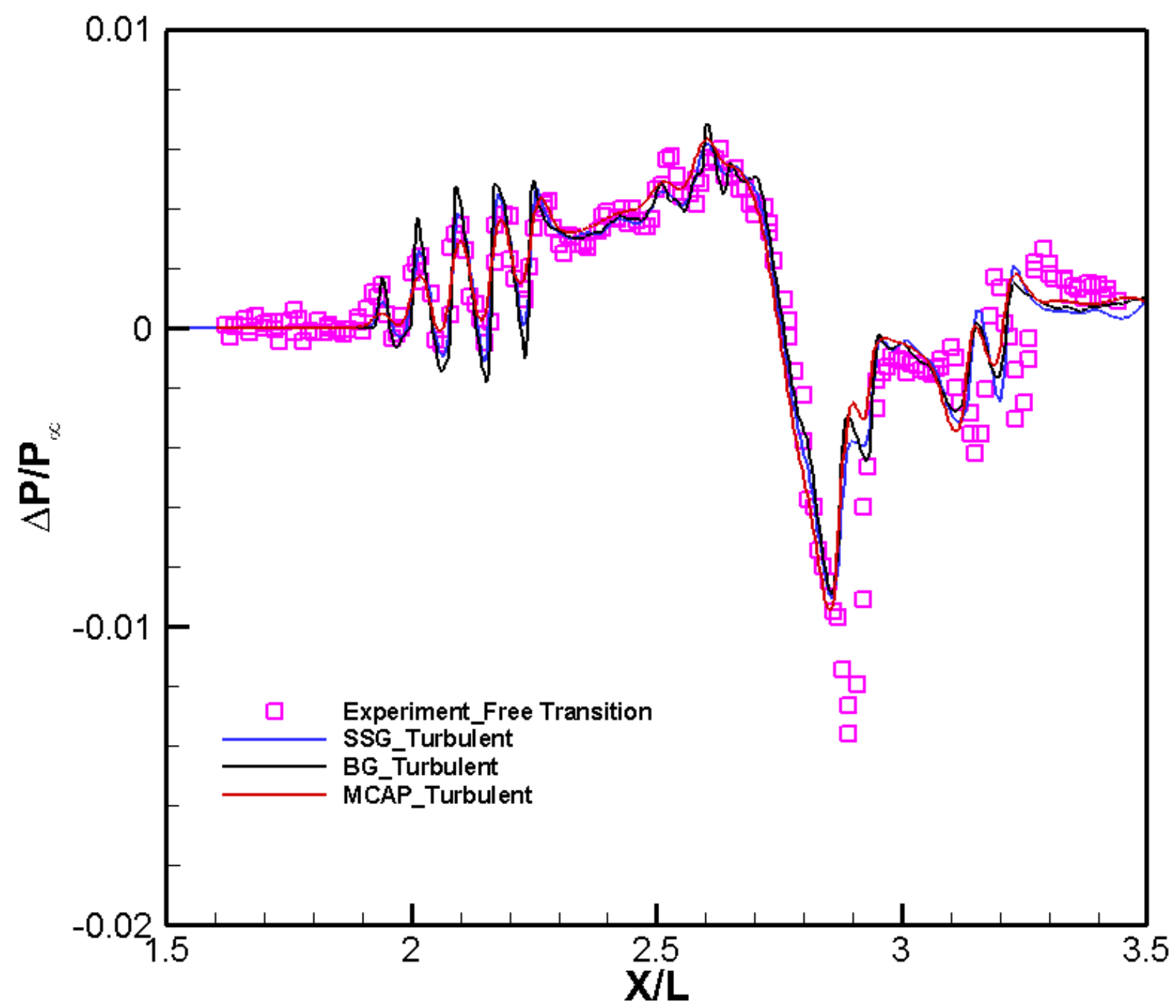

Figure 18. Comparison of experimental data with 35 degrees off-track pressure signatures of grid adaption schemes SSG, MCAP, and BG for turbulent flow condition on the Gulfstream configuration $(H / L=1.756$, Mach number=1.56, $\alpha=0.256$ degrees, $R_{L}=3.86$ million). 


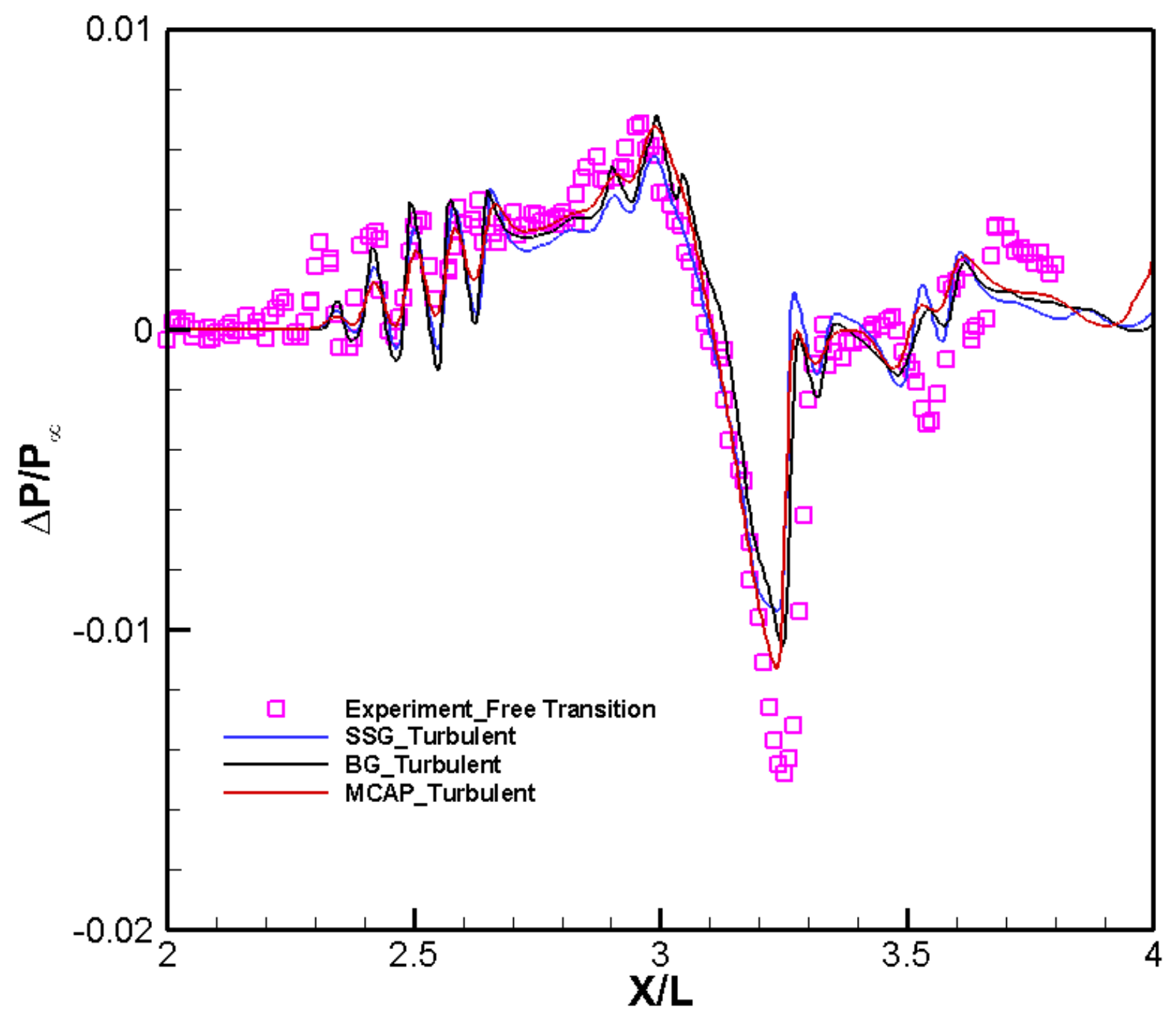

Figure 19. Comparison of experimental data with 53 degrees off-track pressure signatures of grid adaption schemes SSG, MCAP, and BG for turbulent flow condition on the Gulfstream configuration $(H / L=1.829$, Mach number $=1.56, \alpha=0.256$ degrees, $\operatorname{Re}_{\mathrm{L}}=3.86$ million). 


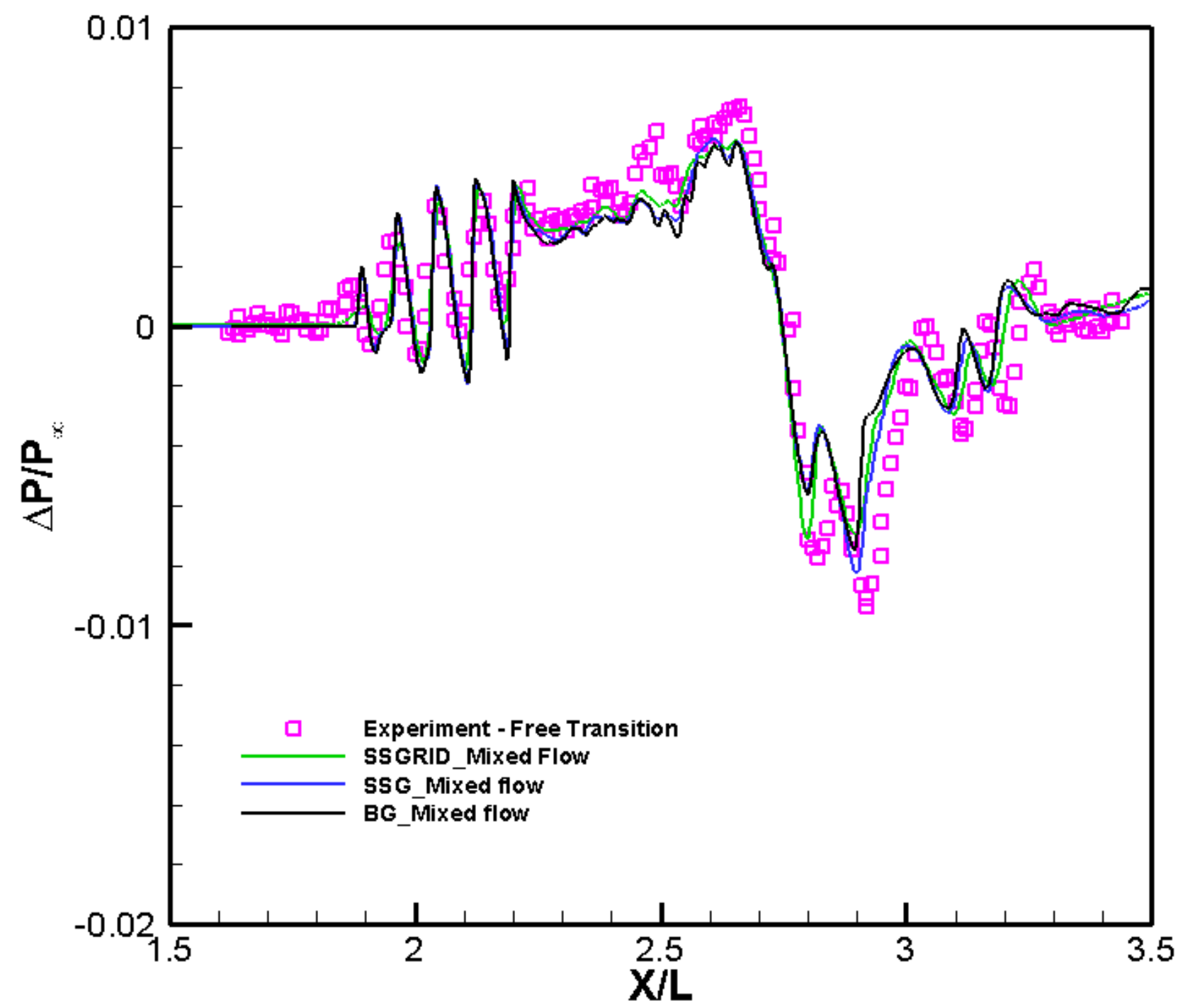

Figure 20. Comparison of experimental data with on-track pressure signatures of grid adaption scheme SSGRID, SSG, and BG for mixed-flow condition on the Gulfstream configuration (wing treated as laminar, $H / L=1.7$, Mach number=1.56, $\alpha=0.256$ degrees, $R_{L}=3.86$ million). 


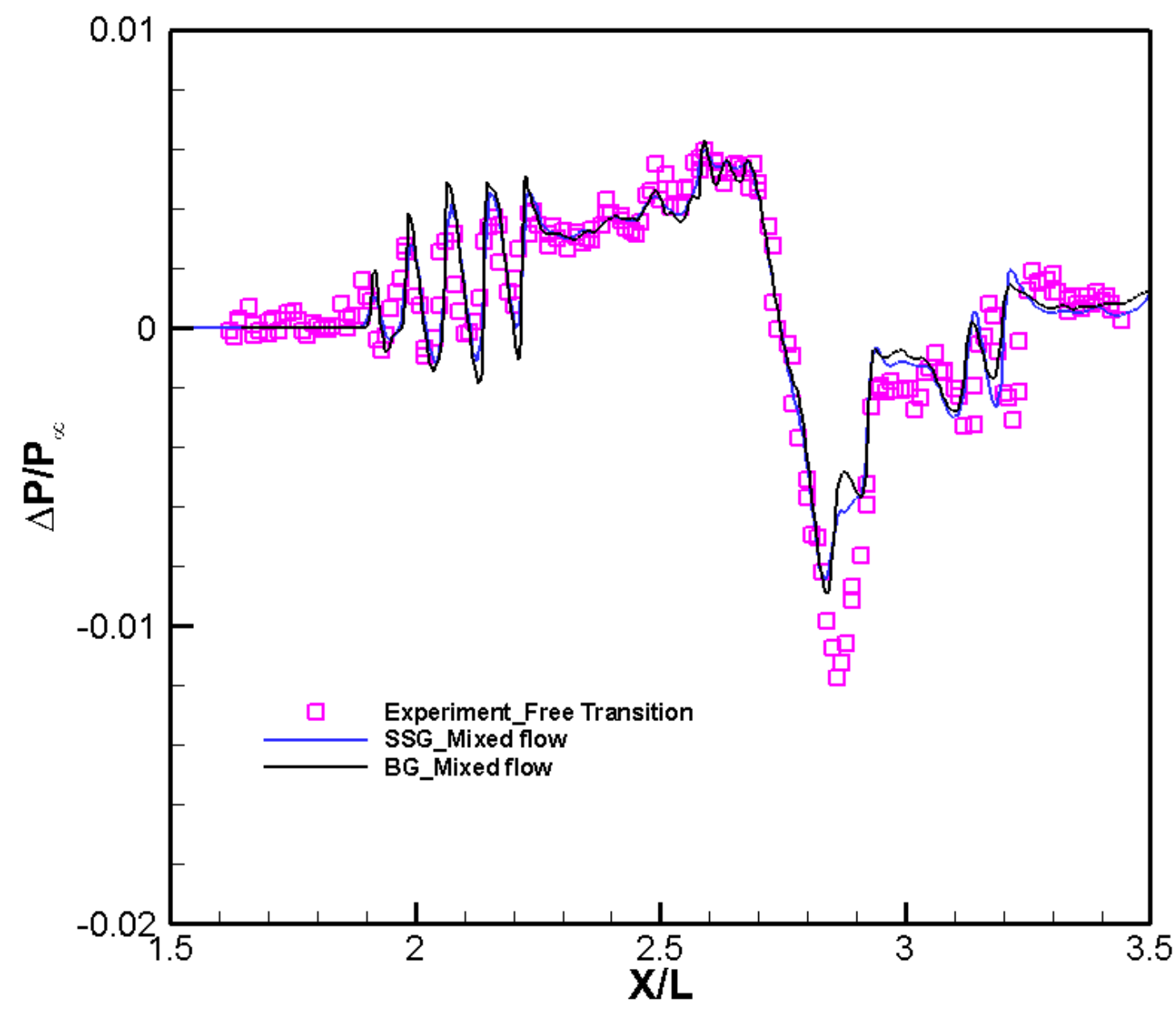

Figure 21. Comparison of experimental data with 25 degrees off-track pressure signatures of grid adaption schemes SSG, and BG for mixed-flow condition on the Gulfstream configuration (wing treated as laminar, $H / L=1.73$, Mach number $=1.56, \alpha=0.256$ degrees, $R_{L}=3.86$ million). 


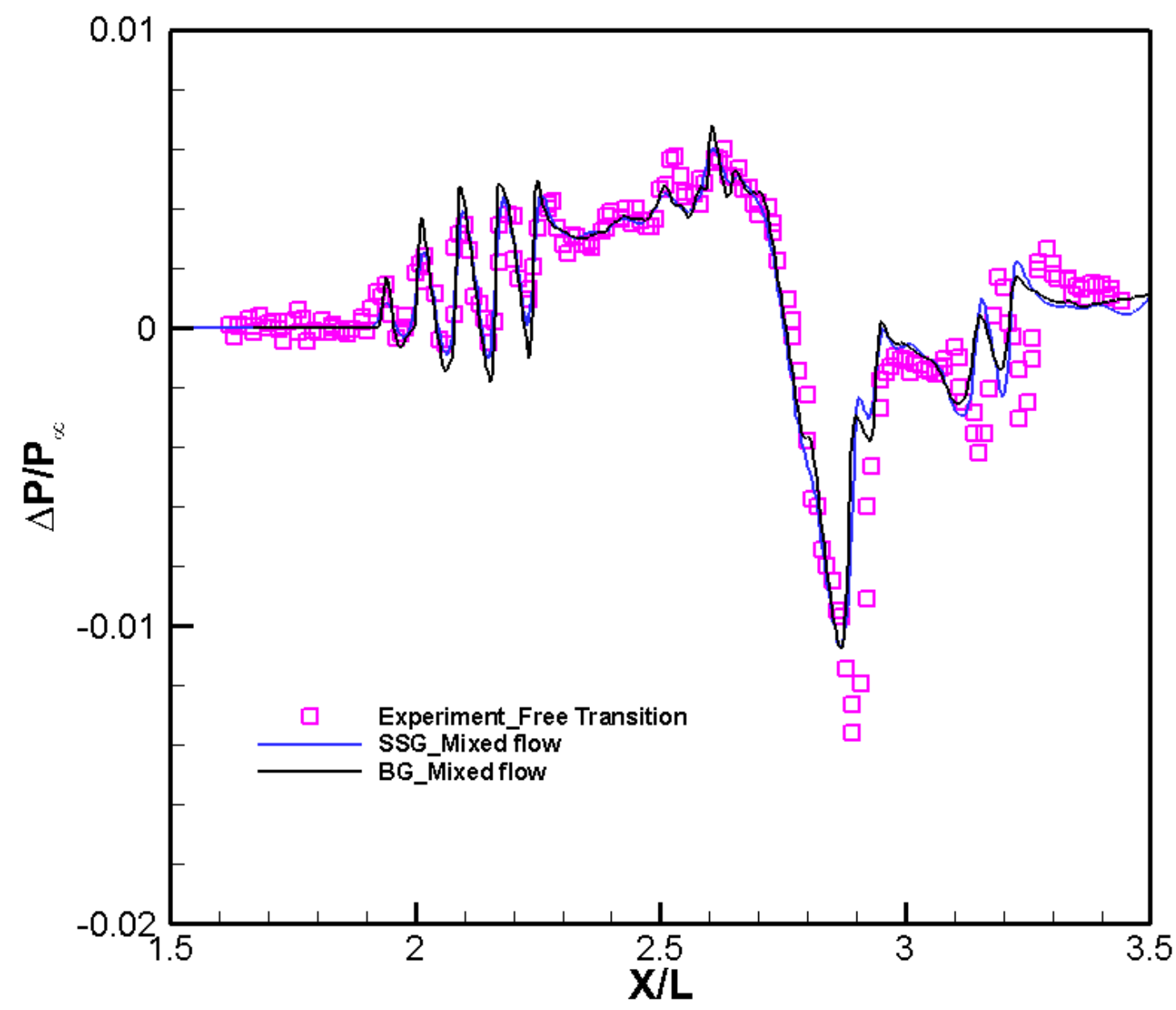

Figure 22. Comparison of experimental data with 35 degrees off-track pressure signatures of grid adaption schemes SSG, and BG for mixed-flow condition on the Gulfstream configuration (wing treated as laminar, $H / L=1.756$, Mach number $=1.56, \alpha=0.256$ degrees, $R_{L}=3.86$ million). 


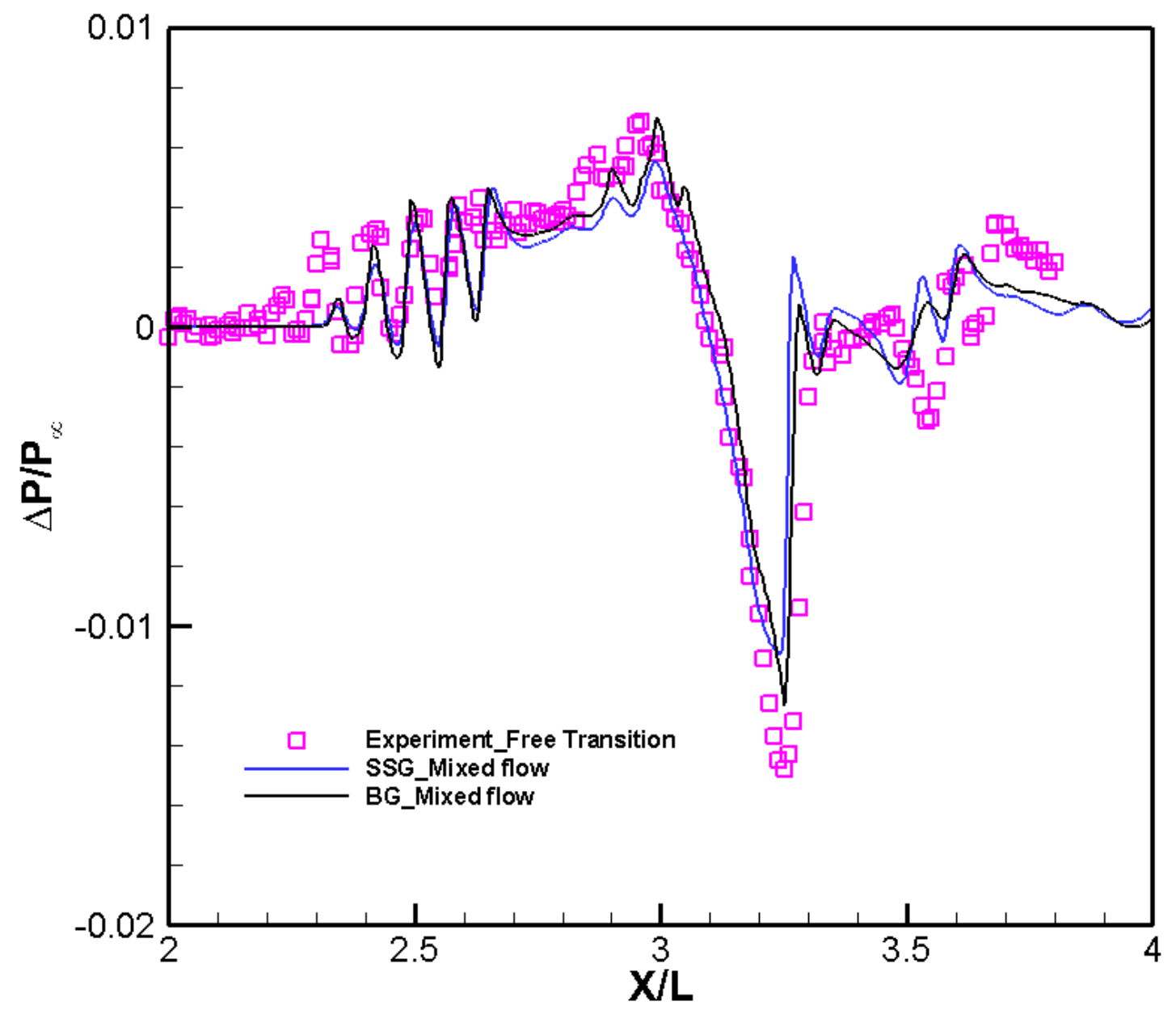

Figure 23. Comparison of experimental data with 53 degrees off-track pressure signatures of grid adaption schemes SSG, and BG for mixed-flow condition on the Gulfstream configuration (wing treated as laminar, $H / L=1.829$, Mach number $=1.56, \alpha=0.256$ degrees, $R_{L}=3.86$ million). 


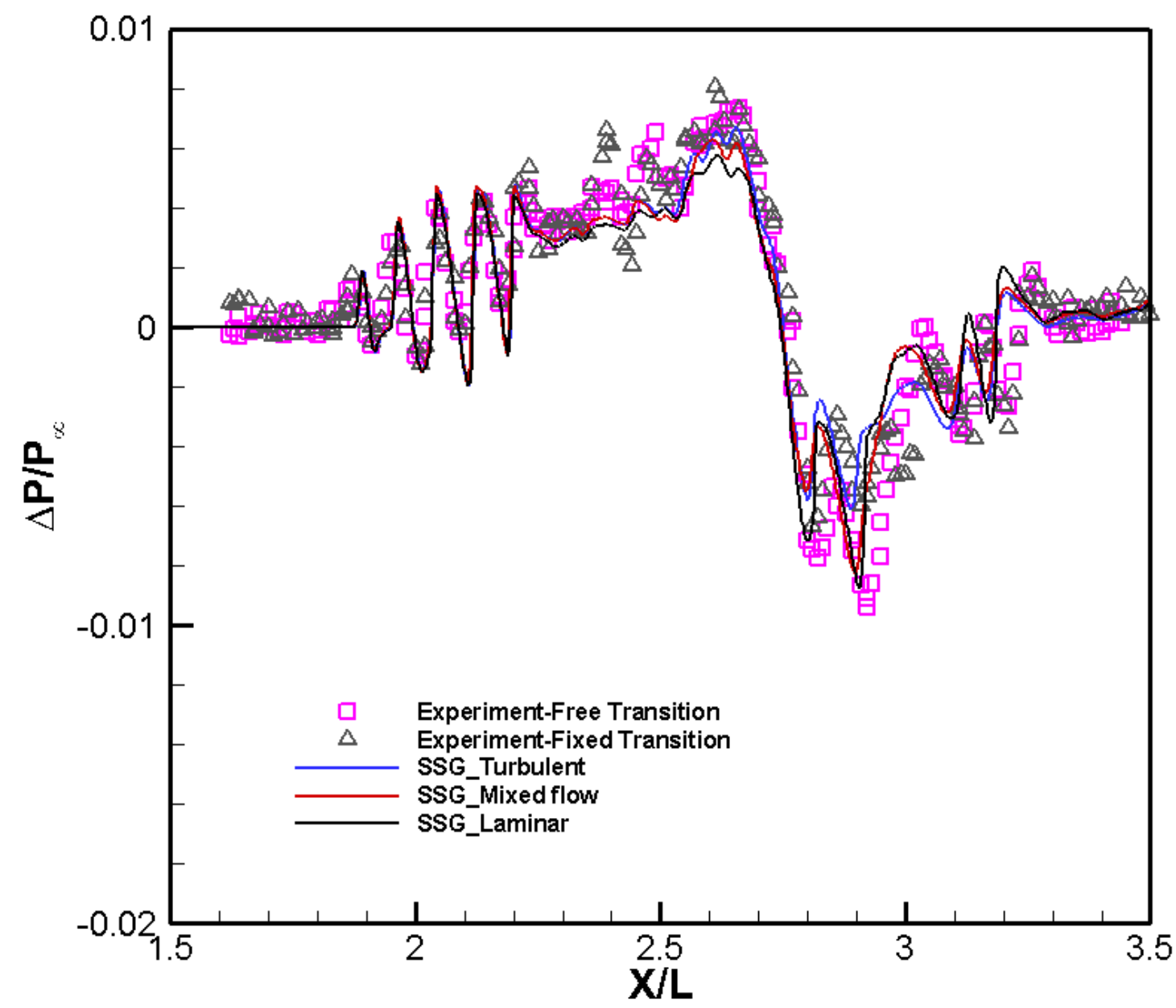

Figure 24. Comparison of experimental data with on-track pressure signatures of grid adaption scheme SSG for different boundary layer states on the Gulfstream configuration $(H / L=1.7$, Mach number=1.56, $\alpha=0.256$ degrees, $\operatorname{Re}_{\mathrm{L}}=3.86$ million). 


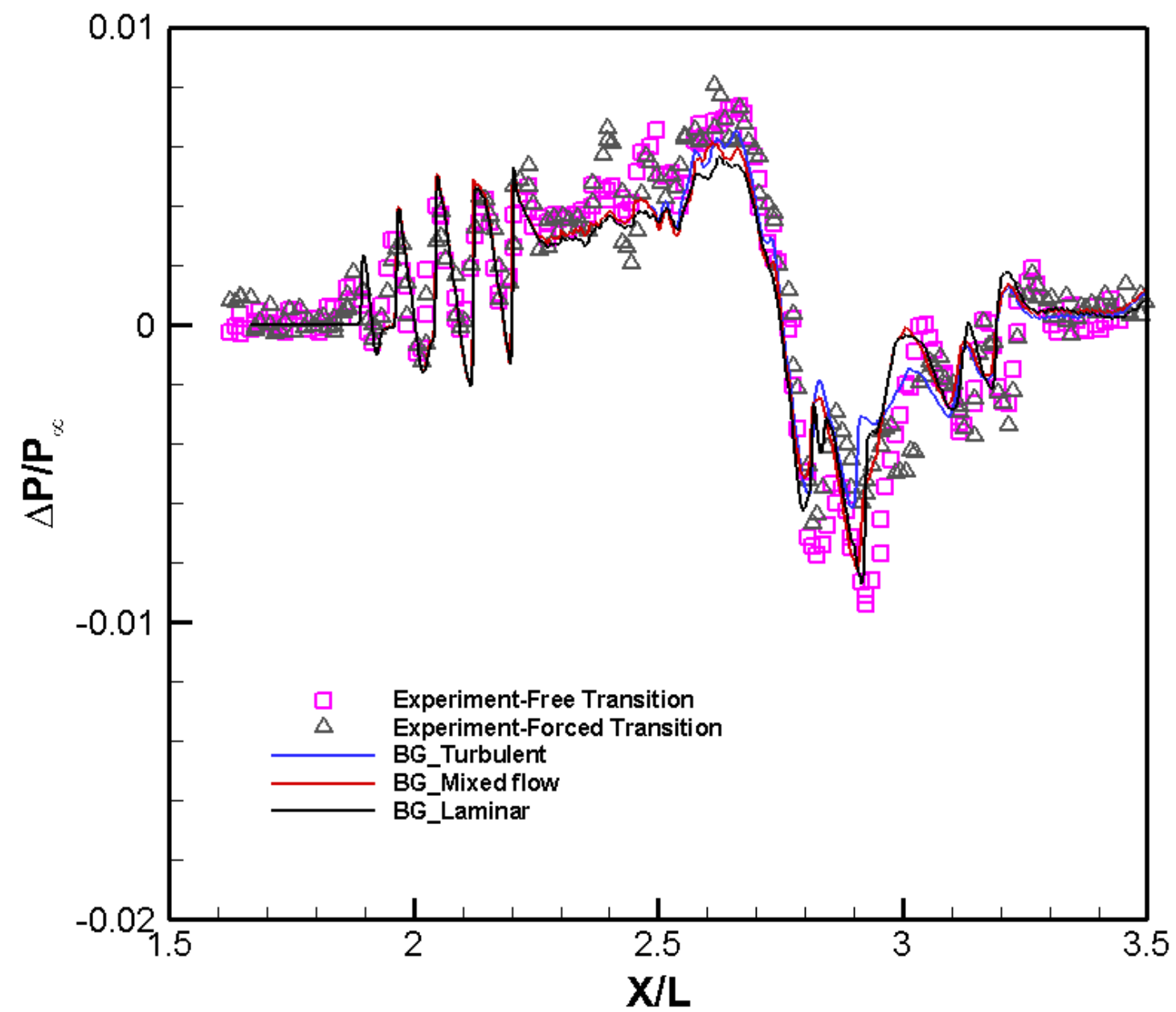

Figure 25. Comparison of experimental data with on-track pressure signatures of grid adaption scheme BG for different boundary layer states on the Gulfstream configuration $(H / L=1.7$, Mach number=1.56, $\alpha=0.256$ degrees, $\operatorname{Re}_{\mathrm{L}}=3.86$ million). 

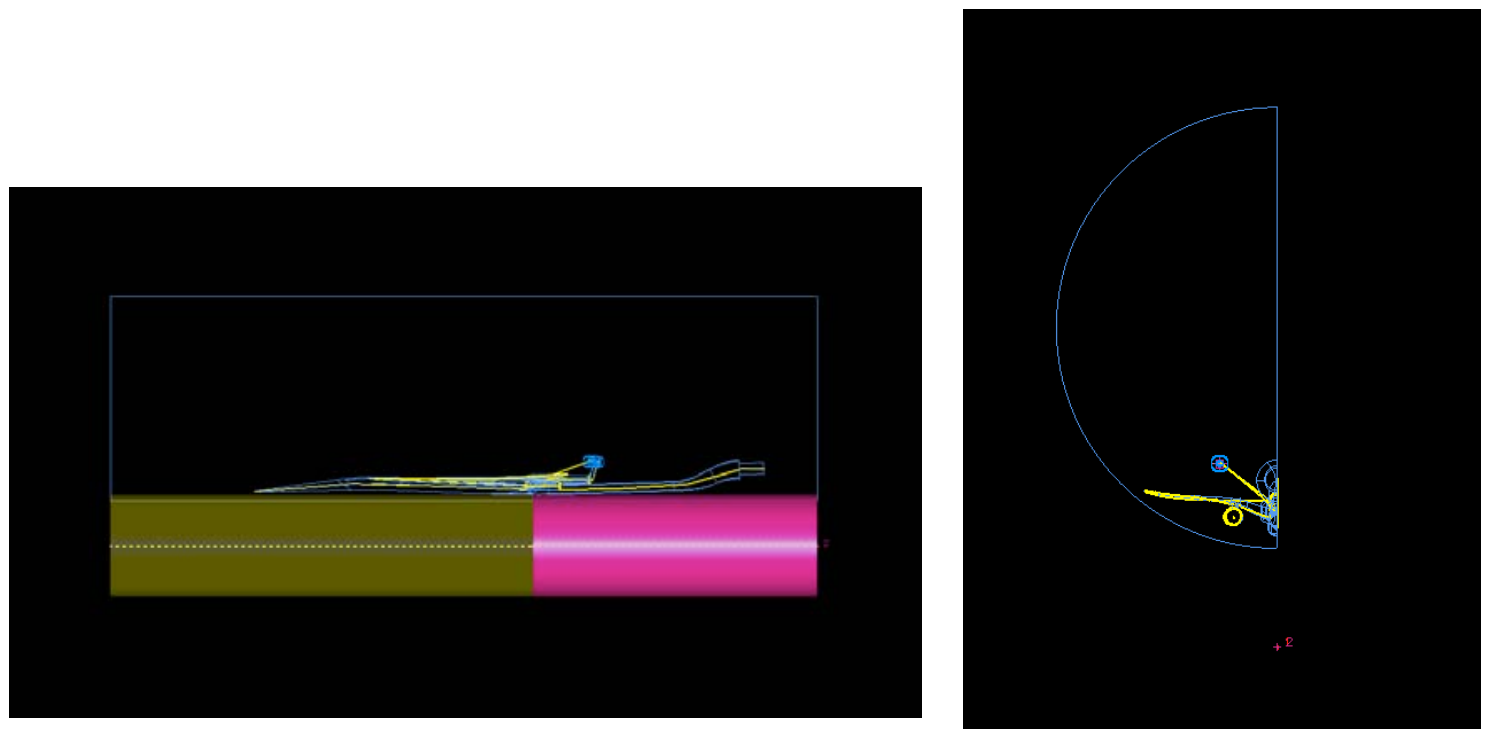

a. Sourcing in GridTool - side view

b. Sourcing in GridTool - rear view

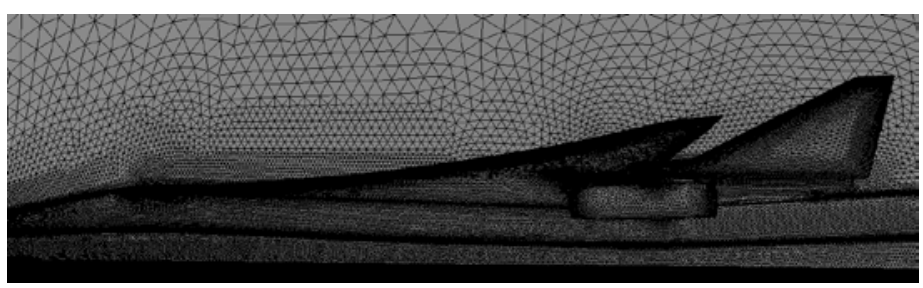

c. Surface grid from VGRID

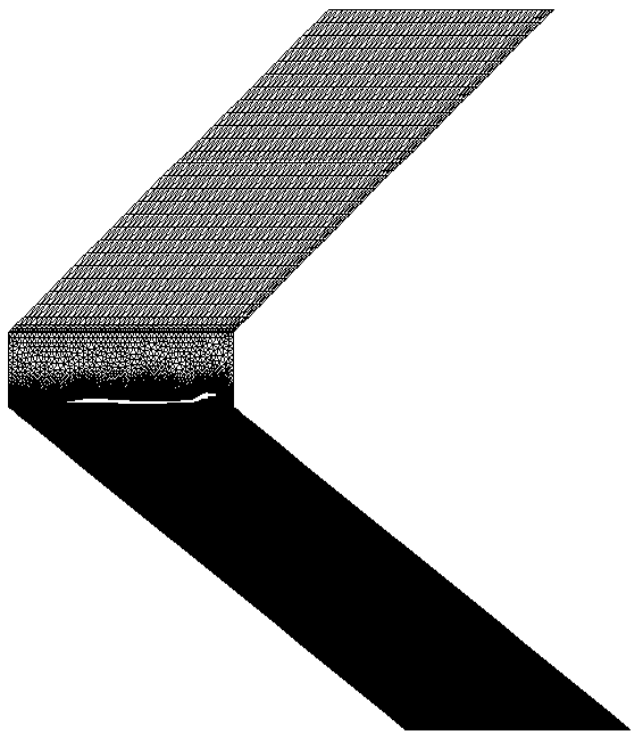

d. Symmetry plane grid after stretching

Figure 26. Grid generation on the Lockheed configuration. 


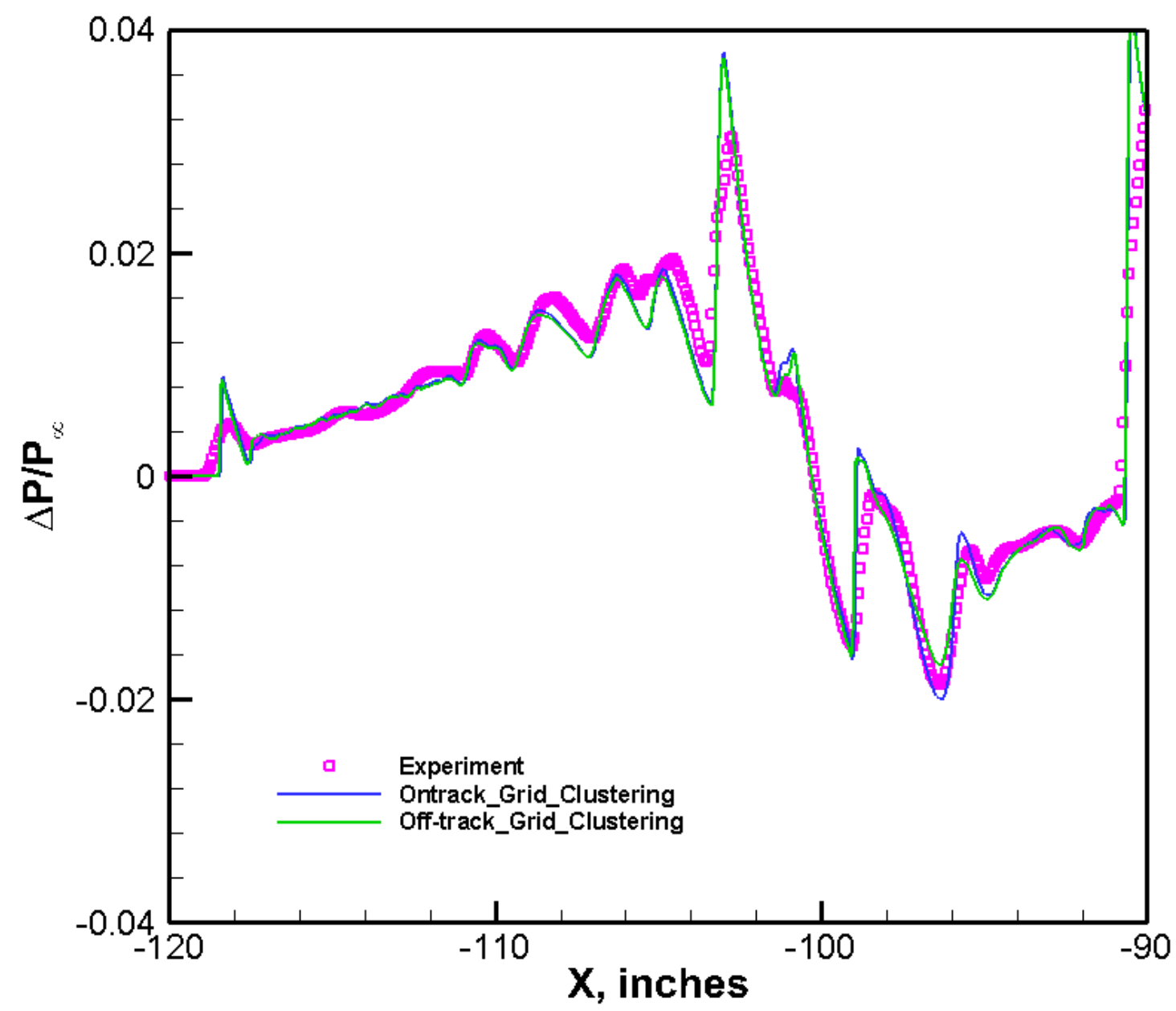

Figure 27. Comparison of experimental data with on-track pressure signatures for the Lockheed Martin configuration for on-track and 40 degrees off-track grid clustering (Mach number=1.6, $\alpha=2.6$ degrees, $\operatorname{Re}_{\mathrm{L}}=8.0$ million, $\left.\mathrm{H} / \mathrm{L}=0.94\right)$. 


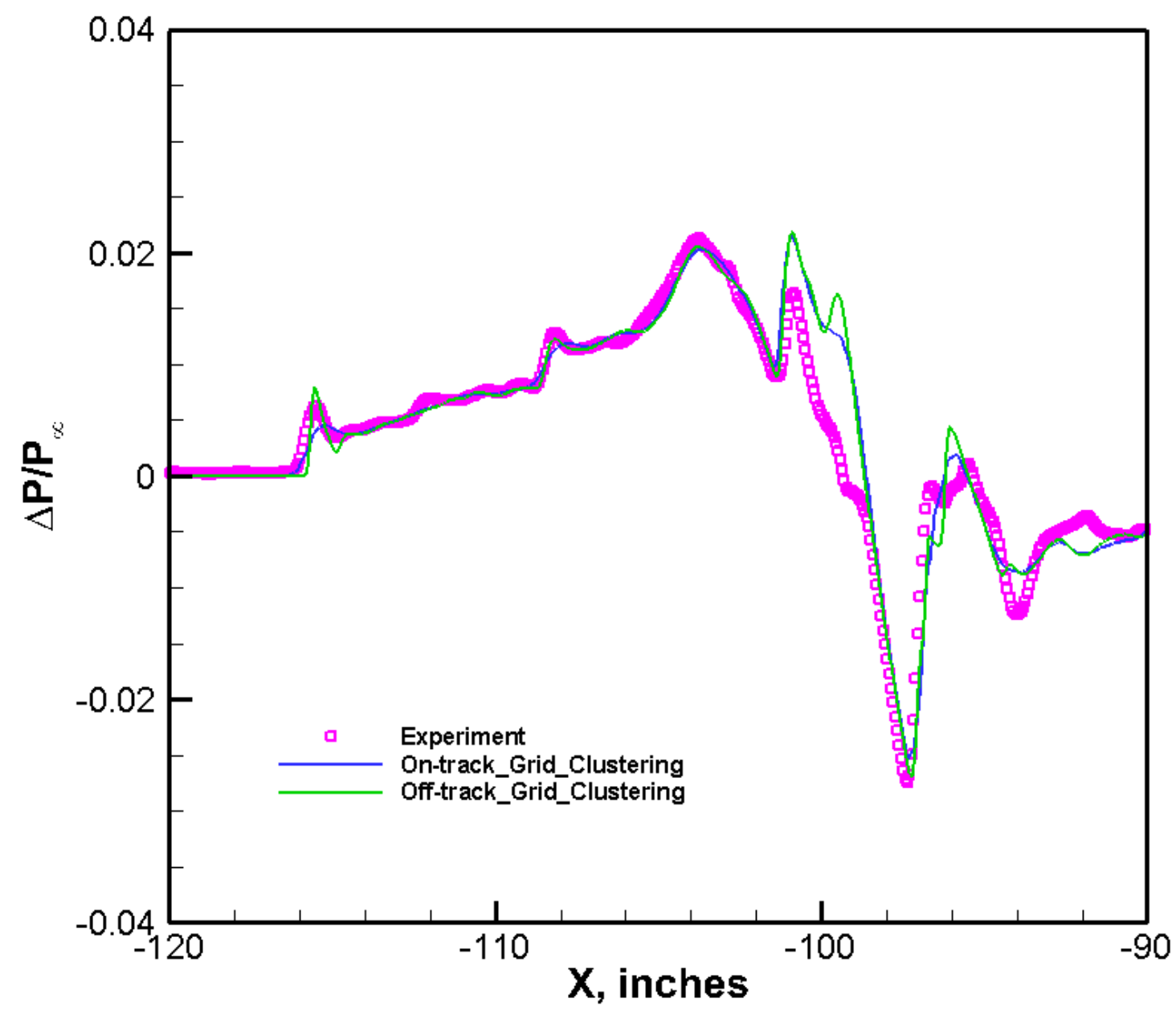

Figure 28. Comparison of experimental data with $\mathbf{4 0}$ degrees off-track pressure signature for the Lockheed Martin configuration for on-track and 40 degrees off-track grid clustering (Mach number=1.6, $\alpha=3.0$ degrees, $\operatorname{Re}_{\mathrm{L}}=8.0$ million, $\left.\mathrm{H} / \mathrm{L}=0.94\right)$. 


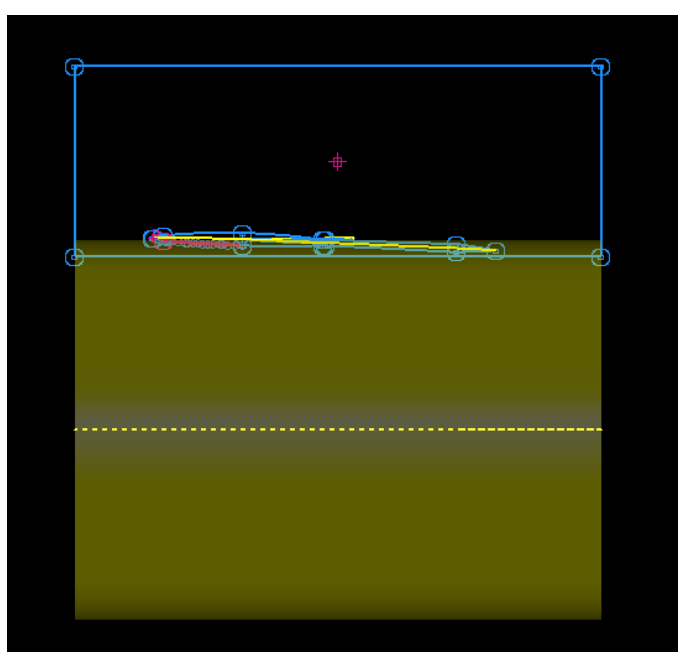

a. Sourcing in GridTool - side view

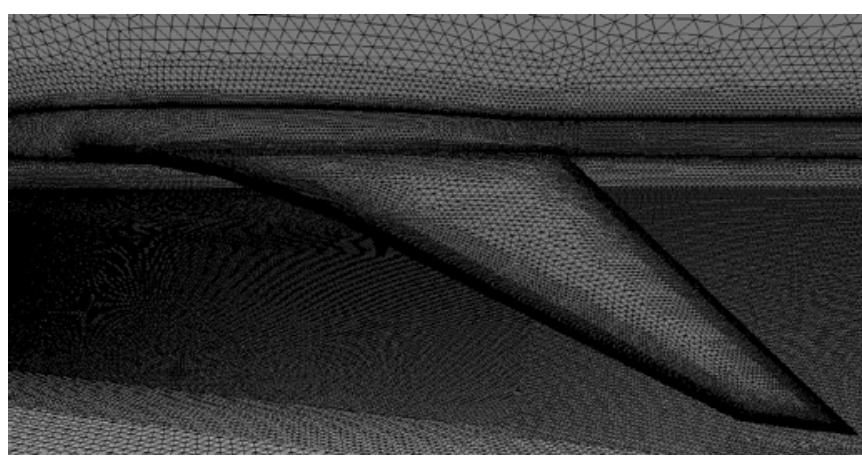

c. Surface grid from VGRID

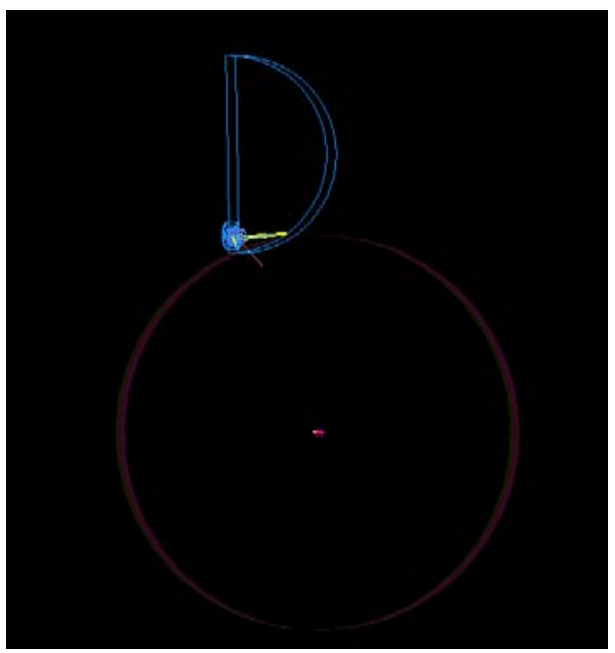

b. Sourcing in GridTool - rear view (slightly tilted to show boom source)

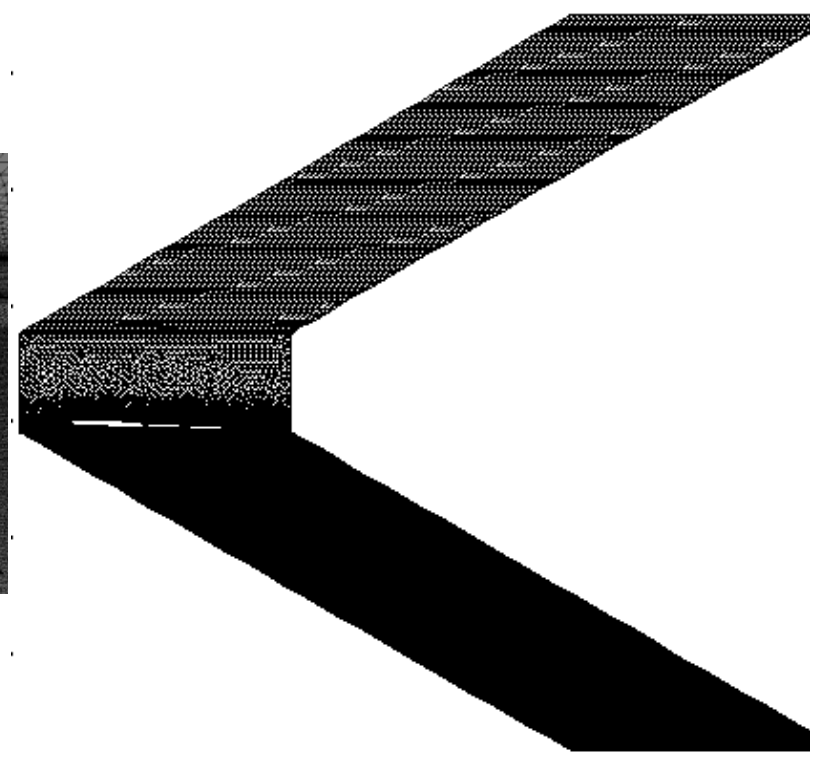

d. Symmetry plane grid after stretching

Figure 29. Grid generation for the BG method - Straight Line Segmented Leading Edge (SLSLE) configuration. 


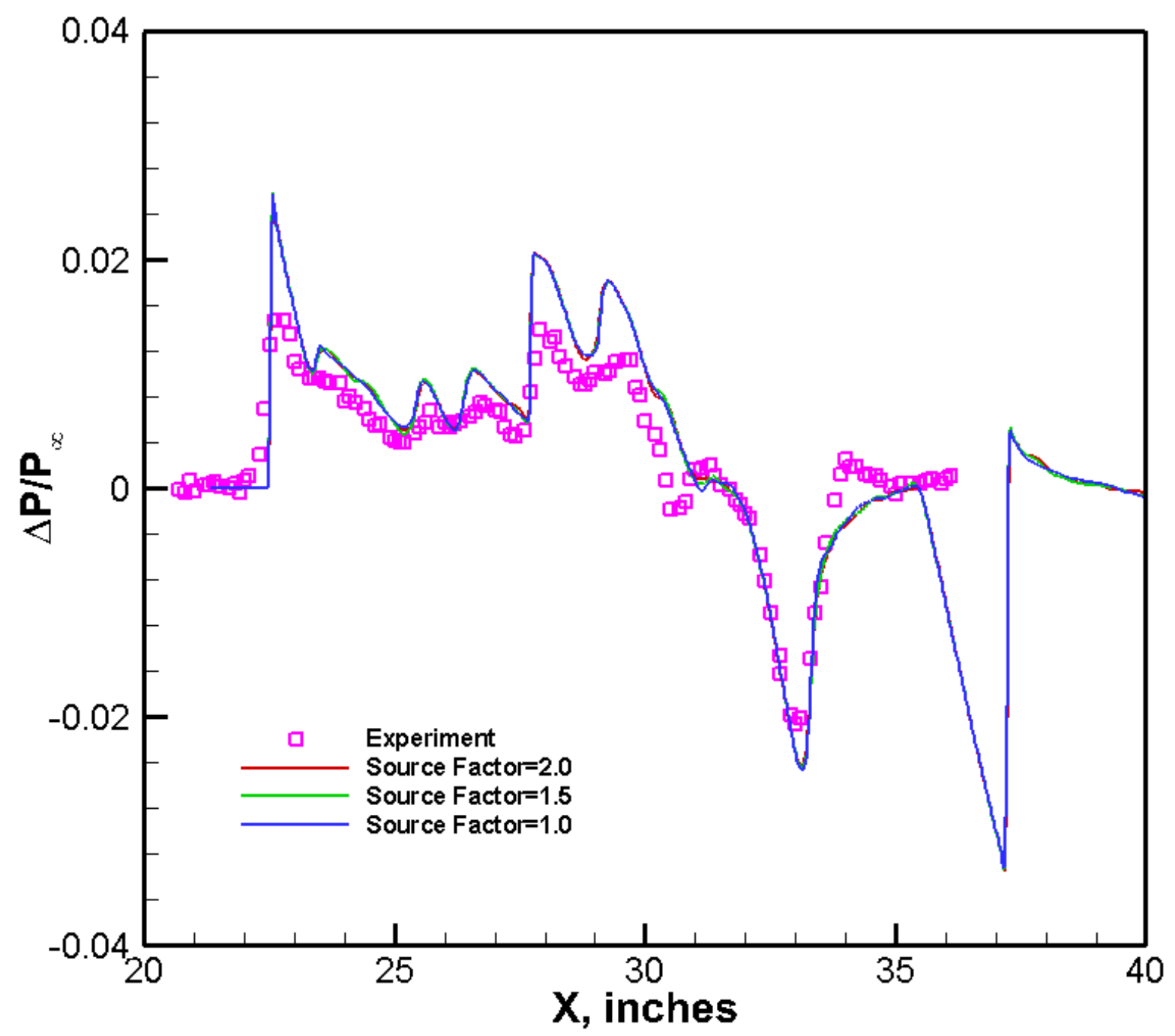

Figure 30. Comparison of experimental data with on-track pressure signature for the SLSLE model for three overall source factors (Mach number=2.0, $\alpha=2.3$ degrees, $R_{L}=1.5$ million, $H / L=1.5$ ). 


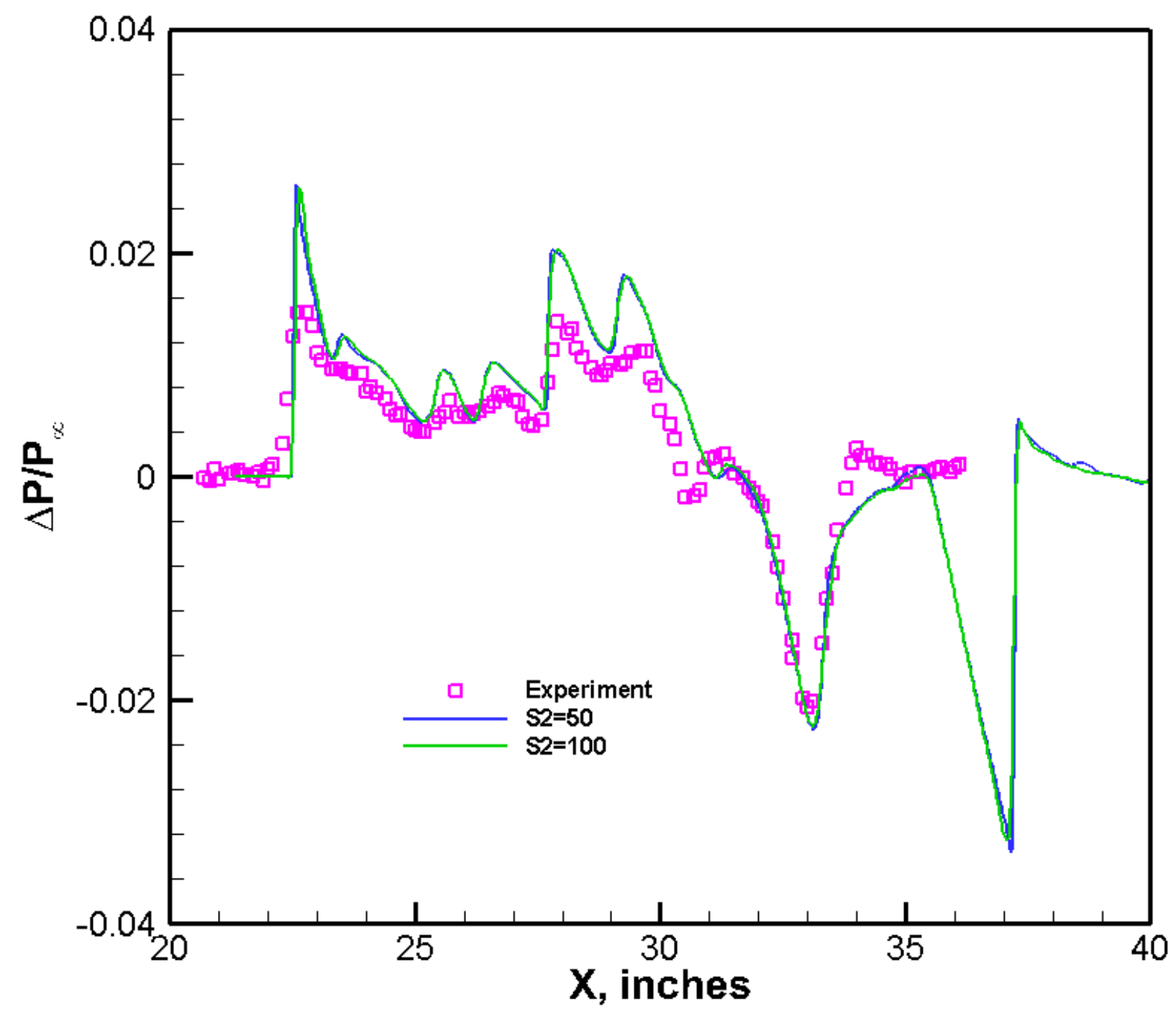

Figure 31. Comparison of experimental data with on-track pressure signature at $H / L=1.5$ for the SLSLE model for two stretching factors (Mach number=2.0, $\alpha=2.3$ degrees, $R_{L}=1.5$ million). 


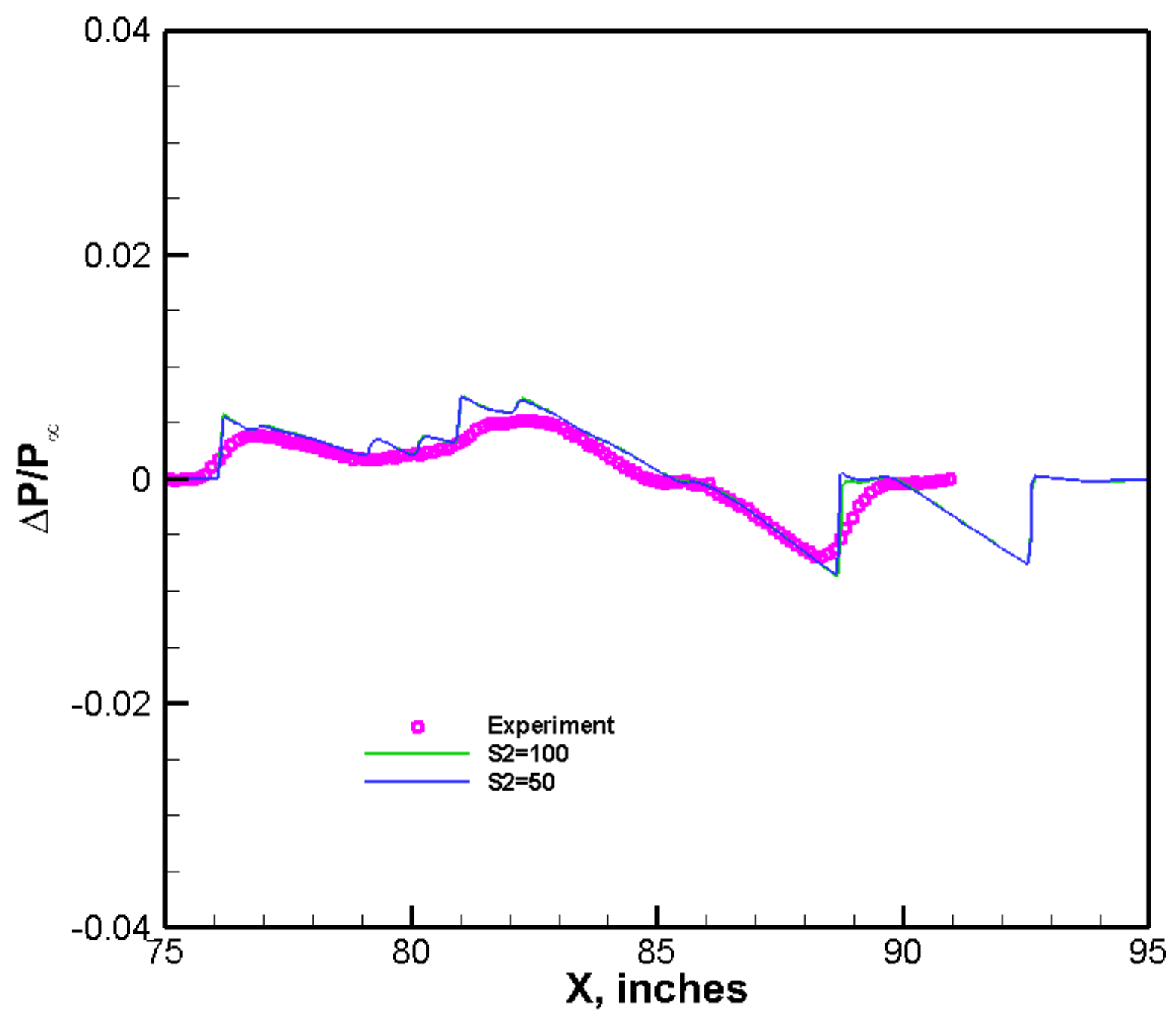

Figure 32. Comparison of experimental data with on-track pressure signature at $H / L=10$ for the SLSLE model for two stretching factors (Mach number=2.0, $\alpha=2.3$ degrees, $\operatorname{Re}_{\mathrm{L}}=1.5$ million). 